\title{
Interstellar polarisation and extinction in the Local Bubble and the Gould Belt
}

\author{
George A. Gontcharov, ${ }^{1,2 \star}$ and Aleksandr V. Mosenkov, ${ }^{3}$ \\ ${ }^{1}$ Department for Management of Science and Technology Development, Ton Duc Thang University, Ho Chi Minh City, Vietnam \\ ${ }^{2}$ Faculty of Applied Sciences, Ton Duc Thang University, Ho Chi Minh City, Vietnam \\ ${ }^{3}$ Central Astronomical Observatory, Russian Academy of Sciences, 65/1 Pulkovskoye chaussee, St. Petersburg, 196140 Russia
}

Accepted 2018 October 29. Received 2018 October 25; in original form 2018 February 16

\begin{abstract}
We analyse an all-sky compilation of thirteen data sources with optical interstellar linear polarisation for 3871 Gaia DR2 and Hipparcos stars within 500 pc, which do not exhibit a considerable intrinsic polarisation. These data are analysed together with five 3D maps and models of the reddening $E(B-V)$. We consider variations of the polarisation degree $P$, position angle $\theta$, and polarisation efficiency $P / E(B-V)$ with Galactic coordinates, distance $R$, and dereddened colour. $P$ shows a maximum at the Gould Belt mid-plane. $P / R$ drops in the Local Bubble by several times defining a boundary of the Bubble at $P=0.1$ per cent. All the data sources of the reddening, except Lallement et al., show a drop of $P / E(B-V)$ in the Bubble. A significant number of stars with a too high $P / E(B-V)$, where the reddening is taken from Arenou et al., Drimmel et al. or Lallement et al., either reject the Serkowski's limit $P / E(B-V)<9.3$ per cent per mag or manifest a systematic underestimation of the reddening by these data sources. The spatial and colour dependent variations of $P$ and $E(B-V)$ outside the Bubble compensate each other, resulting in a nearly constant $P / E(B-V)$. A giant envelope of aligned dust dominates at middle and high latitudes outside the Bubble. The Markkanen's cloud, the North Polar Spur, and some other filaments are parts of this envelope.
\end{abstract}

Key words: ISM: bubbles < Interstellar Medium (ISM), Nebulae - (ISM:) dust, extinction < Interstellar Medium (ISM), Nebulae - ISM: magnetic fields < Interstellar Medium (ISM), Nebulae - ISM: structure < Interstellar Medium (ISM), Nebulae polarization $<$ Physical Data and Processes - (Galaxy:) solar neighbourhood $<$ The Galaxy

\section{INTRODUCTION}

Cumulative interstellar extinction to a star and its reddening are measures of the optical depth of the interstellar dust medium. On the other hand, interstellar polarisation of a star is a measure of the alignment of the dust grains by a strong and regular magnetic field between the observer and the star. Thus, interstellar polarisation, combined with interstellar extinction and reddening, as well as with multi-colour photometry and high-precision distances, offers a possibility to study the local distribution and properties of interstellar dust and Galactic magnetic field.

The position angle of polarisation vectors can be a particularly appropriate tool to map the local Galactic magnetic field (Berdyugin, Piirola \& Teerikorpi 2014). It is generally accepted that this field is directed mainly along the Local

^ E-mail: george.gontcharov@tdt.edu.vn spiral arm, i.e. nearly along $l \approx\left(80^{\circ}, 260^{\circ}\right)$ (Stephens et al. 2011). However, local deviations of the field are known (Leroy 1999) and should be studied in detail.

Interstellar extinction and interstellar polarisation must be separated from intrinsic extinction of a star (usually negligible) and its intrinsic polarisation (sometimes dominating). It is a common practice to determine a value for the interstellar polarisation of a star by taking into account an average or median of the measured polarisation of nearby stars, which do not exhibit a considerable intrinsic polarisation (Cotton et al. 2017). Therefore, some detailed 3D maps of the spatial variations of the polarisation degree and position angle are highly needed. Up to now, there have been made only some initial attempts of creating such maps (Fosalba et al. 2002; Stephens et al. 2011; Santos, Corradi \& Reis 2011) .

Polarisation has been measured for thousands of stars and presented in a dozen of catalogues (Heiles 2000). Some 
previous attempts to combine these data (Leroy 1999; Heiles 2000; Fosalba et al. 2002) have shown that the Local Bubble (or Local Void, or Local Cavity) within nearly 100 pc from the Sun is a space with virtually no polarisation, whereas it is considerable outside the Bubble. The Bubble is known as a region of lower interstellar gas density (Welsh et al. 2010) surrounded by a torus of denser gas known, in its turn, as the Gould Belt. It is believed that the Bubble was formed few Myr ago by one or more supernovae and since then there has been no star formation in it (Abt 2011). In contrast, the Belt is a region of ongoing star formation (Bobylev 2014). The Bubble is opened in the direction of the Galactic halo, forming a 'chimney' nearly perpendicular to the plane of the Belt (Welsh et al. 1999). Being tilted to the Galactic midplane at about $20^{\circ}$, the Belt takes maximal and minimal Galactic latitudes near the directions of the Galactic Centre and anticentre, respectively. The Belt extends up to several hundred parsecs from the Sun.

Despite the agreement on the general structure of the nearby space (see Reis et al. 2011; Gontcharov 2016; Perryman 2009, pp. 324-328), the quantitative characteristics of the medium in the Bubble and the Belt remain contradictory. Beginning with FitzGerald (1968), it is believed that the Bubble has lower dust density, reddening, and extinction in addition to lower gas density (see Reis \& Corradi 2008; Bobylev 2014; Lallement et al. 2014; Frisch et al. 2015; Capitanio et al. 2017). However, in fact, FitzGerald only drew a nearby region with a total reddening $E(B-V)<0.01 \mathrm{mag}$. An increase of the reddening outside the Bubble may be a simple consequence of the natural increase of the dust column density with distance when its volume density is nearly constant. FitzGerald did not show that this volume density and related differential reddening $E(B-V) / R$ is lower near the Sun ( $R$ is the distance). This can only be tested with fairly accurately estimated distances in the postHipparcos era. For example, figure 1 from Lallement et al. (2014) and figure 4 from Capitanio et al. (2017) do show the Bubble as a region with lower dust volume density. However, various definitions of the Bubble by estimating $E(B-V)$ at its boundary are contradictory: $E(B-V)<0.010$ (FitzGerald 1968), $E(B-V)<0.045$ (Frisch 2007), $E(B-V)<$ 0.053 given $E(b-y)<0.04$ and $E(B-V)=1.335 E(b-y)$ (Lallement et al. 2014), and even $E(B-V)<0.055$ given the extinction-to-reddening ratio $R_{\mathrm{V}} \equiv A_{\mathrm{V}} / E(B-V)=3.1$ and $A_{\mathrm{V}}<0.17 \mathrm{mag}$ (Reis et al. 2011). A typical accuracy of all these estimates seems to be $\sigma(E(B-V))>0.02 \mathrm{mag}$ (Gontcharov \& Mosenkov 2017b). With such a high uncertainty, it is still to be confirmed, whether the Bubble has lower dust volume density, $E(B-V) / R$ and $A_{\mathrm{V}} / R$.

The low polarisation, reddening, and extinction inside the Bubble, together with their low fractional accuracy, have led to some contradictory statements. For example, Cotton et al. (2016) noted that 'at 100 pc the extinction is well below that which can actually be measured by photometry', in contradiction to a note by Leroy (1999) that 'The walls of the Bubble [closer than $100 \mathrm{pc}$ ] are fairly well defined when one measures the interstellar absorption'. A comparison of polarisation and reddening for the same stars may solve such contradictions.

Some attempts to give a mean relationship between the polarisation degree and the reddening have been based on the data with either a poor statistics or low accuracy. For ex- ample, Fosalba et al. (2002) provided the mean relationship

$P=3.5 E(B-V)^{0.8}$ per cent,

based on an inhomogeneous compilation of $E(B-V)$, which are precise to only $\sigma(E(B-V))=0.1 \mathrm{mag}$. Moreover, due to a rather sparse distribution of the stars, Fosalba et al. had to derive a single equation for the whole space of several kiloparsecs. A very large scatter of these data (figure 4 from Fosalba et al.) suggests that there is a large variety of such relations in different regions of space. Some of our results are compared with those from Fosalba et al. in Section 3.5.

In previous comparisons of polarisation and reddening since Serkowski, Mathewson \& Ford (1975), the maximum value of the polarisation efficiency $P / A_{\mathrm{V}} \approx 3$, i.e. $P / E(B-V) \approx 9.3$ per cent per mag with $R_{\mathrm{V}}=3.1$ has been found empirically (hereafter Serkowski's limit). However, it is based on a rather poor statistics: for example, Serkowski, Mathewson \& Ford used only less than 300 stars, whereas in recent study Planck Collaboration et al. (2015) used only 206 objects. Stars with $P / E(B-V)>9.3$ per cent per mag tend to be considered as suspected intrinsically polarised stars. However, for most of them no indication of an intrinsic polarisation has been found. It is thus worthwhile to verify the Serkowski's limit and $P / E(B-V)$ variations by use of most reliable all-sky reddening data sources.

Today we have data to make more robust conclusions about the spatial variations and relations of interstellar polarisation and reddening/extinction inside and outside the Bubble. These data include new polarisation measurements, reddening data from new sources based on some recent large all-sky photometric and spectroscopic surveys, as well as more accurate parallaxes and photometry from the Gaia DR2 (Gaia Collaboration et al. 2018a,b,c). Note that Heiles (2000) and Fosalba et al. (2002) are the latest all-sky compilation of the polarisation data and all-sky analysis of their spatial variations, respectively. Thus, the potential of very accurate Gaia parallaxes has not been used.

A new dataset and method of Lallement et al. (2014, hereafter LVV) are especially promising for the nearby space with low reddening. 'One of the main advantages of the present method and of the dataset is the large number of nearby stars for which very low extinctions have been measured' (LVV).

The aim of our study is to compile an all-sky polarisation dataset in- and outside the Bubble, to show this dataset versus the most precise reddening estimates and to study some variations of $P, \theta, E(B-V)$, and $P / E(B-V)$ as some functions of the Galactic coordinates $R, l$, and $b$, and stellar colour.

Besides some global variations, we intend to test some minor assumptions. For example, a higher polarisation has been found for stars of the spectral classes B and M (see Cotton et al. 2016). However, in all the samples under consideration these classes are more luminous and distant. Therefore, the higher polarisation of these classes may be only a consequence of the polarisation versus distance relation (Bailey et al. 2010).

This paper is organised as follows. In the next section, we present polarimetric data and analyse spatial variations of the polarisation degree and position angle. In Section 3, we analyse the polarisation degree together with the redden- 
ing and polarisation efficiency as some functions of Galactic coordinates and dereddened colour of stars. Our conclusions are presented in Section 4.

\section{POLARISATION}

Throughout this work, the polarisation degree $P$ and position angle $\theta$ are given in per cents and degrees, respectively. All measurements of $\theta$ are referred to Galactic coordinates (Stephens et al. 2011).

In order to analyse spatial variations of polarisation and reddening, we have to consider stars with precise parallaxes. This is especially important because all reddening data sources used give reddening as some functions of 3D coordinates. We select Gaia DR2 stars with precise measurements of optical linear polarisation and within $500 \mathrm{pc}$ based on the distances from Bailer-Jones et al. (2018). Also, we select 55 bright stars with precise polarisation measurements, which have precise Hipparcos parallaxes (van Leeuwen 2007) but not included in Gaia DR2. The median fractional parallax uncertainty of the sample is very low: $\sigma(\varpi) / \varpi=0.01$ and, hence, has a negligible contribution to other uncertainties. 21 stars with $\sigma(\varpi) / \varpi>0.2$ are eliminated due to their very uncertain $R$ (Bailer-Jones 2015). The space under consideration includes the Local Bubble and nearly the whole Gould Belt. Yet, a rather sparse distribution of stars with a measured polarisation at the edge of this space does not allow us to detect the outer boundary of the Belt by any characteristic.

We consider optical polarisation measurements from 13 data sources: Heiles (2000) - 1595 independent values in the final sample, Berdyugin, Piirola \& Teerikorpi (2014) - 1290, Santos, Corradi \& Reis (2011) - 451, Leroy (1999) - 350, Berdyugin et al. (2001) - 97, Berdyugin \& Teerikorpi (2002) - 76, Alves \& Franco (2006) - 62, Cotton et al. (2017) - 45, Wills, Wills \& Breger (2011) - 31, Bailey et al. (2010) - 23, Cotton et al. (2016) - 21, Poidevin \& Bastien (2006) - 20, and Andersson \& Potter (2007) - 6 values after the elimination of some values/stars from the sample, as described below. Some stars have several independent values. Therefore, the number of stars under consideration is slightly lower than the number of values.

To consider the polarisation statistics, we de-biased the polarisation degree estimates, following a standard practice (see Cotton et al. 2017), as:

$P_{\text {debiased }}=\left(P^{2}-\sigma(P)^{2}\right)^{1 / 2}$,

as $P>\sigma(P)$ for our sample.

\subsection{Verification of the sample}

In order to clean and verify the polarisation data, we use all the data with $P / \sigma(P)>1.43$. These data together with the reddenings are presented in the master Table 1 available online. After this verification we select the final sample with $P / \sigma(P)>3$ in order to make more robust conclusions. This restriction translates into $\sigma(\theta)<9.55^{\circ}$ following Serkowski (1974).

The polarisation measurements were fulfilled in the $B, V$, SDSS $g^{\prime}$, a wide visual band, or have been scaled to these bands by the authors of these publications.
No author found significant variations of $P$ or $\theta$ within these bands (Berdyugin et al. 2001; Alves \& Franco 2006; Andersson \& Potter 2007; Berdyugin, Piirola \& Teerikorpi 2014; Cotton et al. 2016). Thus, we can combine these data into one sample.

In our sample we excluded stars with intrinsic polarisation known from the literature. These include all Be stars from Yudin (2001), all stars with IR excess from McDonald, Zijlstra \& Watson (2017), except Gaia DR2 3520586071216143488 and Gaia DR2 4076915349748285952 with negligible intrinsic polarisation (Cotton et al. 2017), as well as several stars from other sources.

Also, we exclude 135 stars with a suspected intrinsic polarisation. They are listed in Table 2. The reasons for excluding these stars are the following: some specific features in their spectral classification (e.g. Ap), duplicity, variability, fast rotation, or high proper motion (probably, followed by misidentification). This information is derived from the SIMBAD database (Wenger et al. 2000), the Tycho-2 Spectral Type Catalog (Wright et al. 2003), the Washington Visual Double Star Catalog (Mason et al. 2001), and other sources. Few stars are excluded due to their discrepant degree of polarisation relative to neighbouring stars. These rejected stars are designated in the notes in the Table 2 as having 'Too high polarisation'. Another reason is a disagreement of more than $3 \sigma$ of the stated errors between the independent polarisation measurements for the same star. Such cases are designated in the notes as 'Disagree'. Only 10 stars among them have such a disagreement as the main reason for a suspected intrinsic polarisation.

For the remaining stars with independent measurements (with a disagreement of less than $3 \sigma$ of the stated errors) Fig. 1 and 2 show a comparison of $P$ and $\theta$, respectively, for the pairs of independent measurements. We note that the limitation $0^{\circ}<\theta<180^{\circ}$, by definition, means that, in fact, $\theta \approx 0^{\circ}$ agrees with $\theta \approx 180^{\circ}$. A good agreement, which is evident from Fig. 1 and 2, allows us to compile all the measurements together. We note that some earlier reported disagreement by $\theta$ in different data sources, for example by Alves \& Franco (2006), is due to its ignored or improper conversion from equatorial to Galactic coordinates. 86 stars with an agreement within $1 \sigma$ of the stated errors for $P$ and within $19^{\circ}$ for $\theta$, having $P / \sigma(P)>3$, are listed in Table 3 .

It is likely that our sample still comprises some stars with a considerable but yet unknown intrinsic polarisation. Nevertheless, we believe that this would not alter the general pattern of the variations revealed in our study. We have tested all our results with and without the excluded stars their influence on the obtained results is very small.

The final sample contains 4067 values of $P$ for 3871 stars and 4038 values of $\theta$ for 3842 stars.

The Hertzsprung-Russell (HR) diagram for our final sample is presented in Fig. 3, where $G, G_{B P}$ and $G_{R P}$ are the Gaia DR2 photometric bands. For 78 stars with no or uncertain $G, G_{B P}$, and $G_{R P}$ they are calculated based on $B_{T}$ and $V_{T}$ photometry from Tycho-2 (Høg et al. 2000) by use of their relation from Gaia Collaboration et al. (2018b). We applied the reddening and extinction from Gontcharov (2017, hereafter G17) taking into account the standard extinction law of Cardelli, Clayton \& Mathis (1989) and spatial variations of $R_{\mathrm{V}}$ from its 3D map of Gontcharov (2012a). 67 stars with high $P / E(B-V)$ are shown with the red dia- 

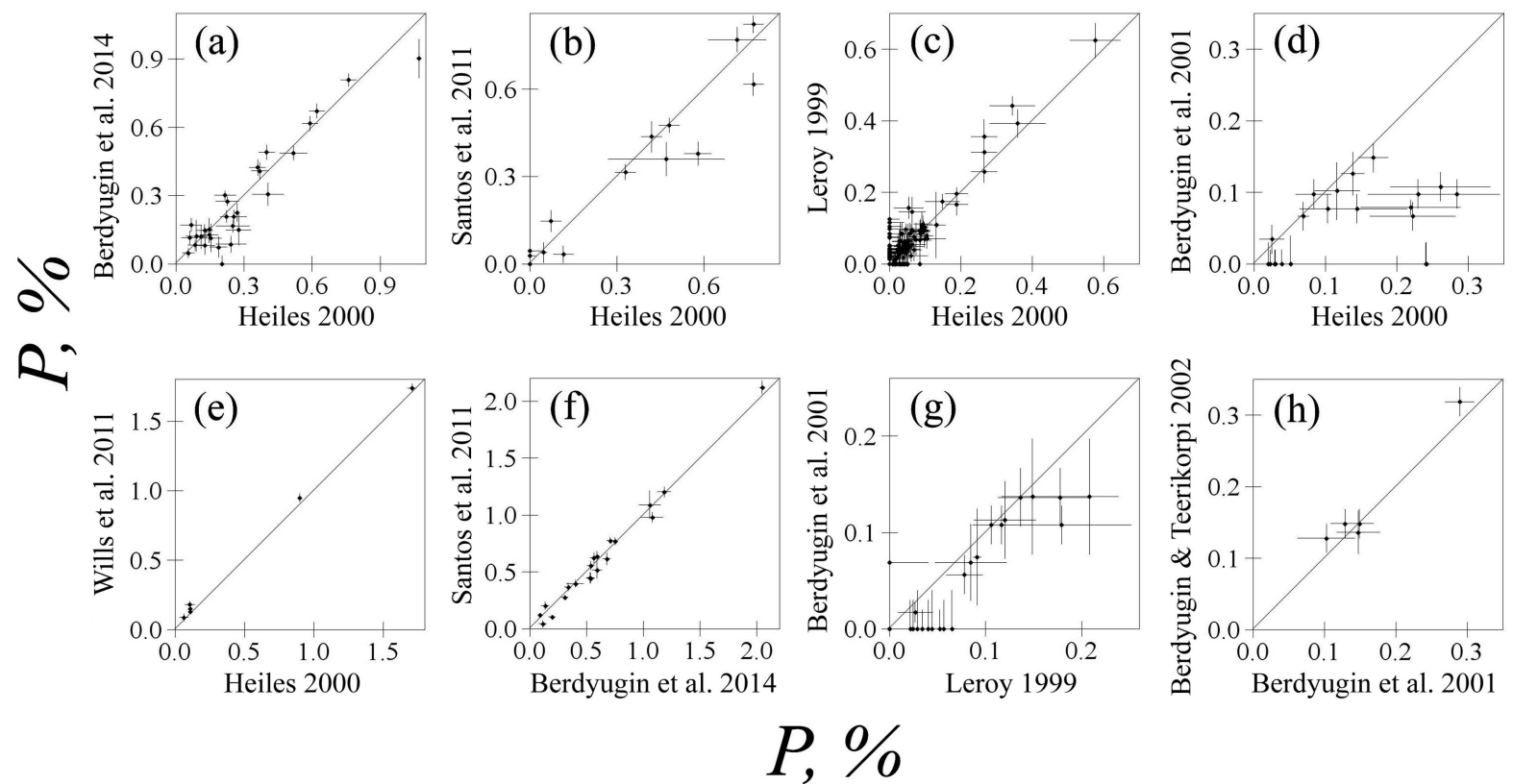

\section{$P, \%$}

Figure 1. Polarisation degree (per cent) for common stars in the pairs of the data sources: (a) Heiles (2000) versus Berdyugin, Piirola \& Teerikorpi (2014), (b) Heiles (2000) versus Santos, Corradi \& Reis (2011), (c) Heiles (2000) versus Leroy (1999), (d) Heiles (2000) versus Berdyugin et al. (2001), (e) Heiles (2000) versus Wills, Wills \& Breger (2011), (f) Berdyugin, Piirola \& Teerikorpi (2014) versus Santos, Corradi \& Reis (2011), (g) Leroy (1999) versus Berdyugin et al. (2001), (h) Berdyugin et al. (2001) versus Berdyugin \& Teerikorpi (2002).

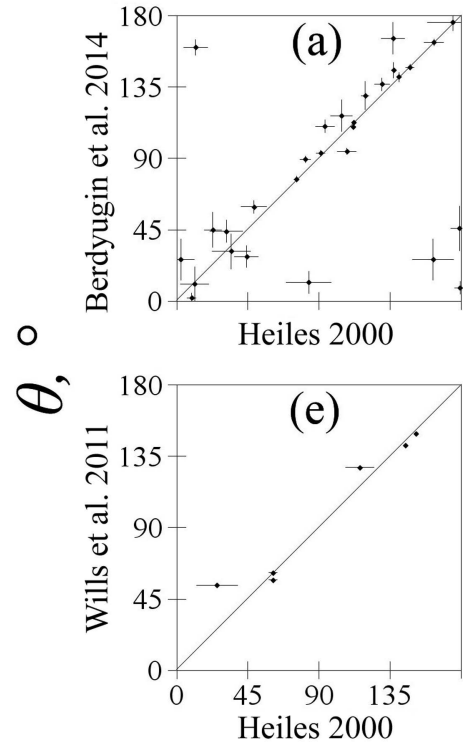

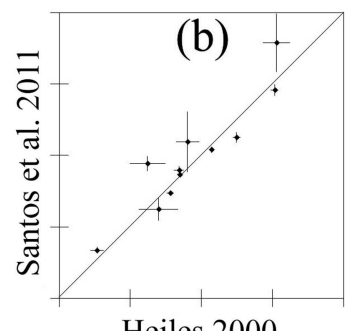

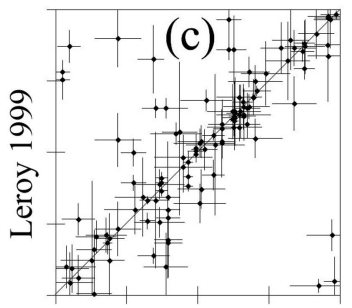

Heiles 2000
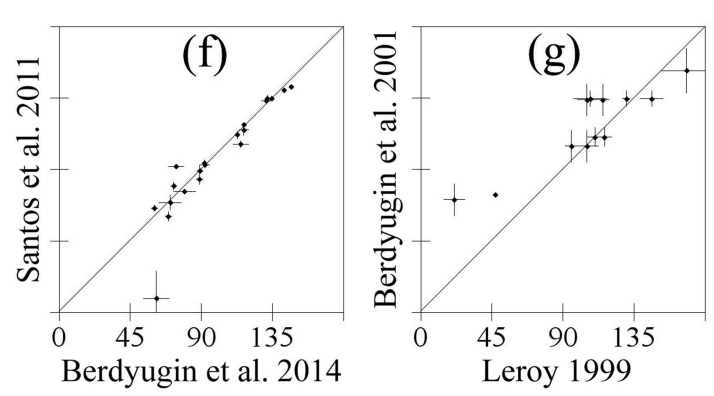

$\theta$

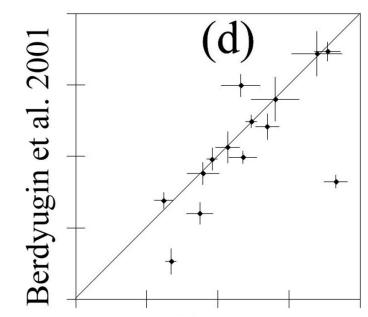

Heiles 2000

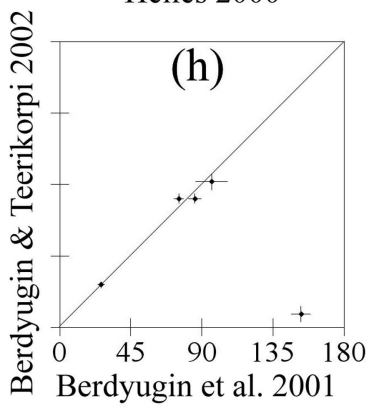

Figure 2. The same as Fig. 1 but for the polarisation position angle (in degrees) for common stars.

monds. They are discussed in Section 3.3. It is seen from Fig. 3 that the giants and early classes of the main sequence are well-represented by our final sample.

The distribution of the sample in the sky by Galactic coordinates is shown in Fig. 4. This distribution is quite representative, yet non-uniform. The same is true for the $3 \mathrm{D}$ distribution of the sample in the Galactic $X Y Z$ coordinates in Fig. 5. The stars with $P<0.1$ and $>0.1$ per cent are shown with the brown and blue symbols. They draw the low and high polarisation in- and outside the Bubble, respectively. 
Table 1. The master table with polarisation and reddening data: the Gaia DR2 source_id or other name, Hipparcos number for stars not found in Gaia DR2, polarisation data source, distance (parsecs), Galactic coordinates (degrees), $P$ and its $\sigma$ (per cents), $\theta$ and its $\sigma$ (degrees), $R_{\mathrm{V}}$, and $E(B-V)$ from G17, G12, AGG, DCL, and LVV. The complete table is available online.

\begin{tabular}{|c|c|c|c|c|c|c|c|c|c|c|c|c|c|c|c|}
\hline DR2 Source_id / Name & HIP & Data source & $R$ & $l$ & $b$ & $P$ & $\sigma(P)$ & $\theta$ & $\sigma(\theta)$ & $R_{\mathrm{V}}$ & G17 & G12 & AGG & DCL & LVV \\
\hline 1002767184492235648 & & Heiles2000 & 108.8 & 156.3675 & 25.5549 & 0.40000 & 0.10000 & 176.7 & 7.2 & 3.10 & 0.066 & 0.061 & 0.016 & 0.011 & 0.004 \\
\hline 1002792679418093696 & & Heiles 2000 & 280.2 & 156.2458 & 25.7459 & 0.60000 & 0.10000 & 176.4 & 4.8 & 2.93 & 0.068 & 0.098 & 0.033 & 0.049 & 0.049 \\
\hline 1008921872627395200 & & Berd2014 & 336.5 & 178.3186 & 41.1850 & 0.08100 & 0.02400 & 102.7 & 8.5 & 2.77 & 0.059 & 0.084 & 0.018 & 0.020 & 0.012 \\
\hline 1009508393361454464 & & Berd2014 & 217.6 & 175.6596 & 42.1903 & 0.03500 & 0.01800 & 72.1 & 14.7 & 3.14 & 0.041 & 0.069 & 0.018 & 0.014 & 0.007 \\
\hline 1009982970068091904 & & Berd2014 & 286.1 & 176.5244 & 40.4675 & 0.08300 & 0.02400 & 106.7 & 8.3 & 2.80 & 0.039 & 0.082 & 0.018 & 0.020 & 0.011 \\
\hline$\cdots$ & $\cdots$ & $\cdots$ & $\cdots$ & $\cdots$ & $\cdots$ & $\cdots$ & $\cdots$ & $\cdots$ & $\cdots$ & $\ldots$ & $\cdots$ & $\cdots$ & $\cdots$ & $\cdots$ & .. \\
\hline
\end{tabular}

Table 2. The 135 excluded stars with a suspected intrinsic polarisation: the Gaia DR2 source_id, G magnitude, distance (parsecs), Galactic coordinates (degrees), spectrum, and notes. The complete table is available online.

\begin{tabular}{lrrrrll}
\hline Gaia DR2 Source_id & $G$ & $R$ & $l$ & $b$ & Spectrum & Note \\
\hline 1053778957742409984 & 4.90 & 101 & 143.5408 & +45.2202 & A1:VpSiSrHg & ET UMa, Variable of alpha2 CVn type \\
1084415852819290112 & 5.99 & 89 & 156.7298 & +31.9494 & A3VpSrSiCrEu & 53 Cam, Variable of alpha2 CVn type, Binary \\
1166857200309419392 & 7.53 & 181 & 10.2360 & +52.0205 & A3p & LV Ser, Variable of alpha2 CVn type \\
1190927159109415552 & 5.29 & 69 & 21.8345 & +47.7510 & A2VpMnEu(Sr) & chi Ser, Variable of alpha2 CVn type, Disagree \\
1197801408889764480 & 5.76 & 54 & 29.6874 & +49.9625 & A8Vam & 22 Ser, Binary \\
$\ldots$ & $\ldots$ & $\ldots$ & $\ldots$ & $\ldots$ & $\ldots$ & $\ldots$ \\
\hline
\end{tabular}

Table 3. The 86 stars with agreed independent polarisation measurements: the Gaia DR2 source_id, G magnitude, distance (parsecs), Galactic coordinates (degrees), weighted mean $P$ and its $\sigma$ (per cents), weighted mean $\theta$ and its $\sigma$ (degrees). The complete table is available online.

\begin{tabular}{lrrrrrrrr}
\hline Gaia DR2 Source_id & $G$ & $R$ & $l$ & $b$ & Mean $P$ & $\sigma(P)$ & Mean $\theta$ & $\sigma(\theta)$ \\
\hline 1163440089969099264 & 8.61 & 218 & 9.0317 & 51.1356 & 0.265 & 0.011 & 137.4 & 1.2 \\
1250169120491310720 & 4.33 & 140 & 12.2481 & 75.4886 & 0.106 & 0.008 & 131.0 & 2.2 \\
1301081662819876480 & 8.03 & 338 & 44.8817 & 39.2163 & 0.268 & 0.019 & 163.2 & 2.1 \\
1501901345598817792 & 6.79 & 120 & 98.4798 & 70.7932 & 0.125 & 0.017 & 93.1 & 4.2 \\
2455241320597417856 & 4.40 & 84 & 157.0765 & -75.1550 & 0.019 & 0.005 & 93.4 & 6.9 \\
$\ldots$ & $\ldots$ & $\ldots$ & $\ldots$ & $\ldots$ & $\ldots$ & $\ldots$ & $\ldots$ & $\ldots$ \\
\hline
\end{tabular}

\subsection{Spatial variations of polarisation degree}

The data under consideration are not enough to create a detailed 3D map of spatial variations of $P$ and $\theta$. Yet, in Table 4 we provide a result of our study: a low resolution map where the median of $P$ is given as a function of the Galactic octant, latitude, and distance range, i.e. for 32 Galactic sectors and 4 distance ranges of $0-85,85-200,200-300$, and 300-500 pc.

Due to the natural variations of the medium in every spatial cell and the lack of data, the accuracy of this map is limited. This map would help to get a preliminary, first estimate of interstellar $P$ for a star.

The value $P=0.1$ per cent can be accepted as a boundary between the regions with low and high polarisation (the same value was accepted by Leroy (1999)). Typically, the former are within 85 pc, i.e. nearly inside the Bubble, while the latter are outside. Few exceptions are: (i) in the $4^{\text {th }}$ octant, $0^{\circ}<b<+45^{\circ}$, several stars within $64<R<85$ pc have a high $P>0.1$ per cent and, hence, constitute a rather nearby wall of the Bubble; (ii) several high latitude 'chimneys' with low $P<0.1$ per cent are evident; (iii) in the $5^{\text {th }}$ octant, $0^{\circ}<b<+45^{\circ}$ and $6^{\text {th }}$ octant, $-45^{\circ}<b<0^{\circ}$, there are two low latitude elongations of the Bubble up to 200 or 300 pc with low $P<0.1$ per cent (interpreted by Leroy 1999 as a possible 'chimney' to another bubble).

Table 4 shows that the variations of $P$ with $R$ are more noticeable than the ones with $l$ and $b$, which are discussed in Section 3.5. Fig. 6 shows the variations of (a) $P$ and (b) $P / R$ with $R$ for all $l$ and $b$. Despite a large scatter of these data, Fig. 6 confirms our conclusions from Table 4: after a sharp increase of $P$ within nearly 100 pc, i.e. within the Bubble and its walls, outside it $P$ increases with $R$ gradually. 


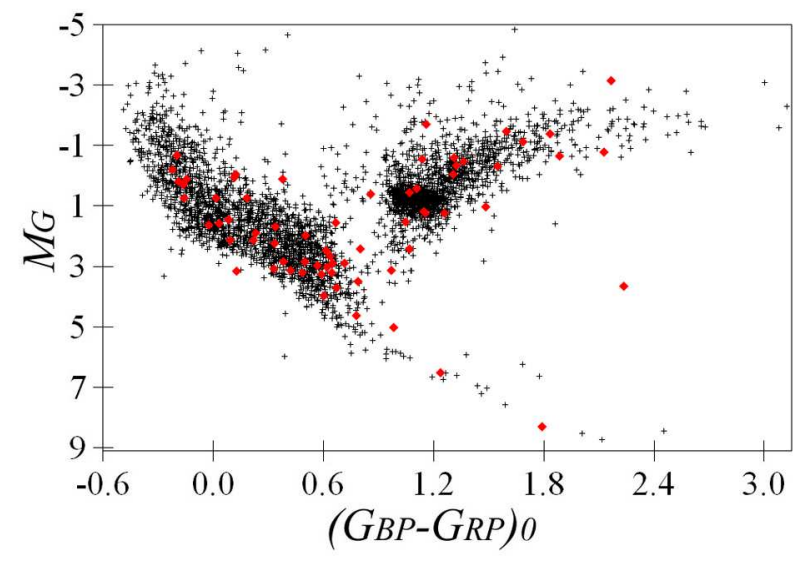

Figure 3. The HR diagram for the final sample. The 67 stars with $P / E(B-V)>9.3$ per cent per mag from all the reddening data sources are shown with the red diamonds.

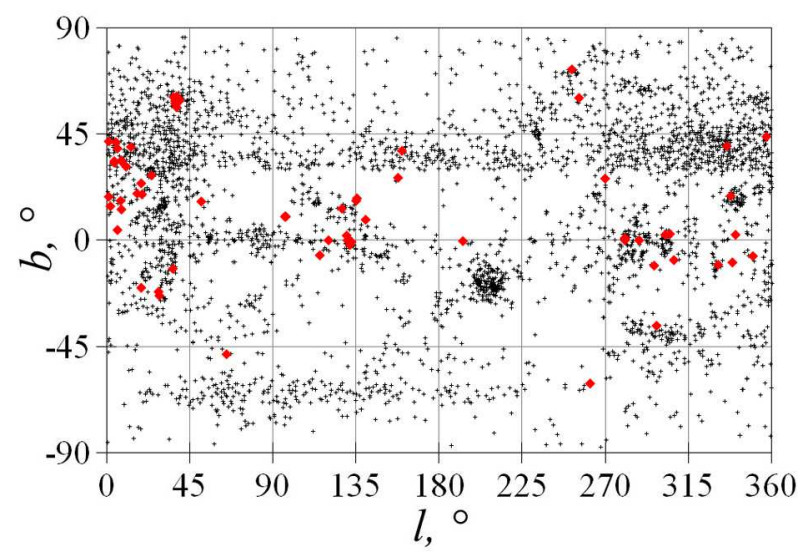

Figure 4. The distribution of the final sample in the sky in Galactic coordinates. The 67 stars with $P / E(B-V)>9.3$ per cent per mag from all the reddening data sources are shown with the red diamonds.

Fig. 6 (b) shows a drop of the average $P / R$ in- w.r.t. outside the Bubble from 1.5 to 0.25 per cent per kpc, i.e. by about 6 times. The maximum of $P / R$ at $R \approx 150 \mathrm{pc}$ and its minimum at $R \approx 450$ pc may suggest a different balance of polarisation and depolarisation of stellar radiation in these three distance ranges: $R<150,150<R<450$, and $R>450$ pc. We note that 57 of 67 stars with $P / E(B-V)>9.3$ per cent per mag from all the reddening data sources, shown with the open diamonds in Fig. 6, are within $98<R<314$ pc, i.e. not far from the maximum of $P / R$. These stars are discussed in Section 3.3.

In Fig. 7 we show three slices of the space under consideration: (a) $X$ versus $Y$ with $-60<Z<60$ pc, (b) $X$ versus $Z$ with $-60<Y<60 \mathrm{pc}$, and (c) $Y$ versus $Z$ with $-60<X<60$ pc. The stars with $P<0.1,0.1<P<0.33,0.33<P<1$, and $P>1$ per cent are shown by different symbols. The regions with these different levels of $P$ are shown by shades of grey. The darkest tone approximately shows the Local Bubble in agreement with its other descriptions, for example with the Na I volume density of $\log n_{\mathrm{Na}}=-9.1 \mathrm{~cm}^{-3}$ iso-contour from Welsh et al. (2010) shown in Fig. 7 (a) as the black curve on the $X Y$ plot. In agreement with Table 4, Fig. 7 shows
Table 4. The median $P$ (per cent) as a function of the Galactic octant from 1 to 8 , latitude (degrees) and distance range (parsecs).

\begin{tabular}{|c|c|c|c|c|c|}
\hline \multirow[t]{3}{*}{ Octant } & \multirow[t]{3}{*}{ Latitude } & \multicolumn{4}{|c|}{$P$, per cent } \\
\hline & & \multicolumn{4}{|c|}{ Distance range, parsecs } \\
\hline & & $0-85$ & $85-200$ & $200-300$ & $300-500$ \\
\hline 1 & $b>+45$ & 0.06 & 0.19 & 0.25 & 0.24 \\
\hline 1 & $0<b<+45$ & 0.09 & 0.51 & 0.49 & 0.58 \\
\hline 1 & $-45<b<0$ & 0.09 & 0.31 & 0.44 & 0.73 \\
\hline 1 & $b<-45$ & 0.01 & 0.08 & 0.12 & 0.08 \\
\hline 2 & $b>+45$ & 0.00 & 0.13 & 0.11 & 0.11 \\
\hline 2 & $0<b<+45$ & 0.00 & 0.17 & 0.18 & 0.19 \\
\hline 2 & $-45<b<0$ & 0.09 & 0.18 & 0.23 & 0.31 \\
\hline 2 & $b<-45$ & 0.09 & 0.14 & 0.17 & 0.18 \\
\hline 3 & $b>+45$ & 0.06 & 0.08 & 0.11 & 0.11 \\
\hline 3 & $0<b<+45$ & 0.03 & 0.18 & 0.25 & 0.50 \\
\hline 3 & $-45<b<0$ & 0.05 & 0.15 & 1.07 & 1.22 \\
\hline 3 & $b<-45$ & 0.02 & 0.11 & 0.23 & 0.19 \\
\hline 4 & $b>+45$ & 0.00 & 0.09 & 0.10 & 0.10 \\
\hline 4 & $0<b<+45$ & 0.22 & 0.21 & 0.14 & 0.18 \\
\hline 4 & $-45<b<0$ & 0.08 & 0.30 & 0.56 & 0.71 \\
\hline 4 & $b<-45$ & 0.02 & 0.08 & 0.14 & 0.18 \\
\hline 5 & $b>+45$ & 0.04 & 0.08 & 0.08 & 0.11 \\
\hline 5 & $0<b<+45$ & 0.05 & 0.10 & 0.08 & 0.11 \\
\hline 5 & $-45<b<0$ & 0.09 & 0.19 & 0.22 & 0.30 \\
\hline 5 & $b<-45$ & 0.03 & 0.10 & 0.16 & 0.15 \\
\hline 6 & $b>+45$ & 0.04 & 0.13 & 0.12 & 0.11 \\
\hline 6 & $0<b<+45$ & 0.08 & 0.13 & 0.14 & 0.19 \\
\hline 6 & $-45<b<0$ & 0.03 & 0.03 & 0.14 & 0.28 \\
\hline 6 & $b<-45$ & 0.04 & 0.05 & 0.08 & 0.11 \\
\hline 7 & $b>+45$ & 0.00 & 0.15 & 0.15 & 0.17 \\
\hline 7 & $0<b<+45$ & 0.01 & 0.15 & 0.25 & 0.29 \\
\hline 7 & $-45<b<0$ & 0.05 & 0.16 & 0.40 & 0.51 \\
\hline 7 & $b<-45$ & 0.03 & 0.04 & 0.10 & 0.08 \\
\hline 8 & $b>+45$ & 0.04 & 0.19 & 0.24 & 0.31 \\
\hline 8 & $0<b<+45$ & 0.09 & 0.30 & 0.52 & 0.54 \\
\hline 8 & $-45<b<0$ & 0.07 & 0.16 & 0.35 & 0.55 \\
\hline 8 & $b<-45$ & 0.02 & 0.10 & 0.17 & 0.23 \\
\hline
\end{tabular}

the Bubble as a nearly spherical central cavity with several low latitude elongations and at least two wide 'chimneys' to the Galactic halo in the northern $2^{\text {nd }}$ and southern $4^{\text {th }}$ quadrants.

Fig. 6 and 7, together with Table 4, may solve some contradictions in the description of this space. For example, the note "the interstellar contribution [to polarisation] is not likely to be much more than $150 \mathrm{ppm}$ [i.e. 0.015 per cent] even at 100 pc' (Cotton et al. 2016) seems to be controversial. Now we see that $P=0.1$ per cent is a typical value at 100 pc.

The black line in Fig. 7 (b) shows the orientation of the mid-plane of the Gould Belt. It is tilted to the Galactic mid-plane by an angle of about $19^{\circ}$. The Belt is rotated so that its part with the highest $b \approx+19^{\circ}$ is at $l \approx 15^{\circ}$, whereas its part with the lowest $b \approx-19^{\circ}$ is at $l \approx 195^{\circ}$ (Gontcharov 2009, 2012b). It is evident from both Fig. 7 and Table 4 that polarisation is higher along the Gould Belt, i.e. in the $1^{s t}$ and $8^{t h}$ octants above the Galactic mid-plane and in the $4^{\text {th }}$ and $5^{\text {th }}$ octants below the plane. 


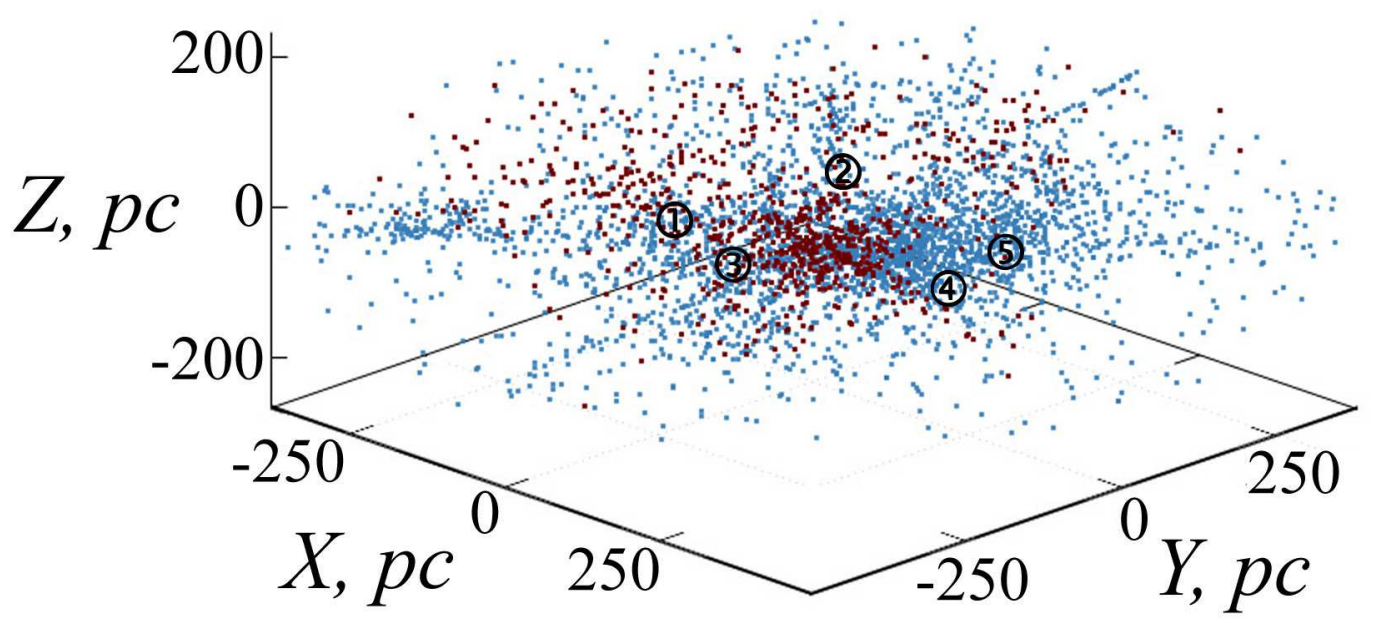

Figure 5. The 3D distribution of the sample in the Galactic $X Y Z$ coordinates. The stars with $P<0.1$ and $>0.1$ per cent are shown with the brown and blue symbols. The position of some bright stars is shown by the following digits: $1-$ Betelgeuse, $2-$ Polaris, $3-$ Canopus, 4 - Antares, and 5 - Enif.
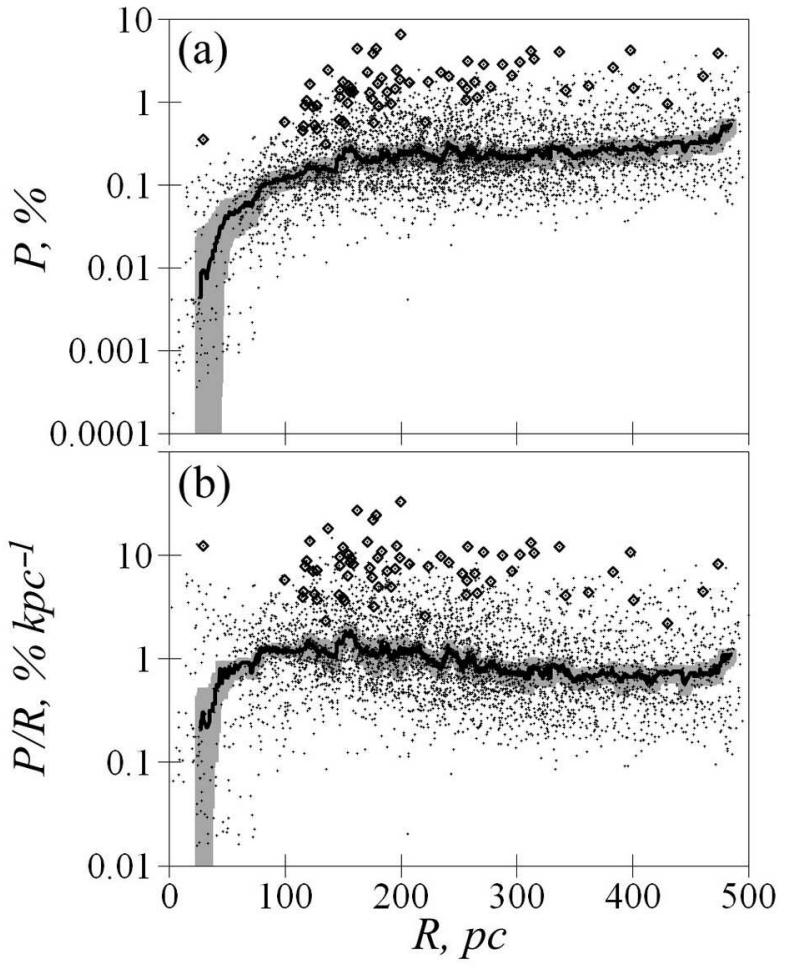

Figure 6. $R$ (parsecs) versus (a) $P$ (per cent) and (b) $P / R$ (per cent per kiloparsec). The 67 stars with $P / E(B-V)>9.3$ per cent per mag from all the reddening data sources are shown with the open diamonds. The black curves with the grey error belts are the moving medians over 101 points.

Fig. 8 shows the distribution of the subsample limited by $R<300 \mathrm{pc}$ along the coordinates (a) $Z$ and (b) $\zeta$, the distance from the mid-plane of the Gould Belt in the direction perpendicular to this plane. $\zeta$ is an analogue of $Z$ in the reference frame of the Belt. The definition of $\zeta$ is given by Gontcharov (2009):

$\zeta=R(\cos \gamma \sin b-\sin \gamma \cos b \cos l)$, where $\gamma \approx 19^{\circ}$ is the tilt of the Belt to the Galactic midplane. Fig. 8 confirms that a maximum $P$ coincides with the mid-plane of the Gould Belt $(\zeta=0 \mathrm{pc})$, but not with the Galactic mid-plane $(Z=0 \mathrm{pc})$. The shift of the distribution in Fig. 8 (a) to positive $Z$ is explained by a combination of the growth of $P$ to both the mid-plane of the Belt and to the Galactic Centre.

We note a good agreement of our results with those from Medan \& Andersson (2018) (see their Table 1), who have used the data from Berdyugin, Piirola \& Teerikorpi (2014) and found a sharp increase of $P$ ('a wall of the Bubble') at rather high $b>+30^{\circ}$ between the Galactic longitudes $350^{\circ}$ and $30^{\circ}$, where Fig. 7 (b) shows plenty of green squares and purple snowflakes. Apparently, this increase is due to the influence of the Gould Belt, which rises up to $b=+19$ deg and dominates within $+10<\mathrm{b}<+40$ deg at these longitudes. Yet, we note that our sample contains too little stars in this northern part of the Belt, i.e. near the upper part of the black line in the $X Z$ plot of Fig. 7. This may lead to an underestimation of the importance of the Belt as a dust container and a domain of the spatial variations of $P$. More polarisation observations are needed here. 


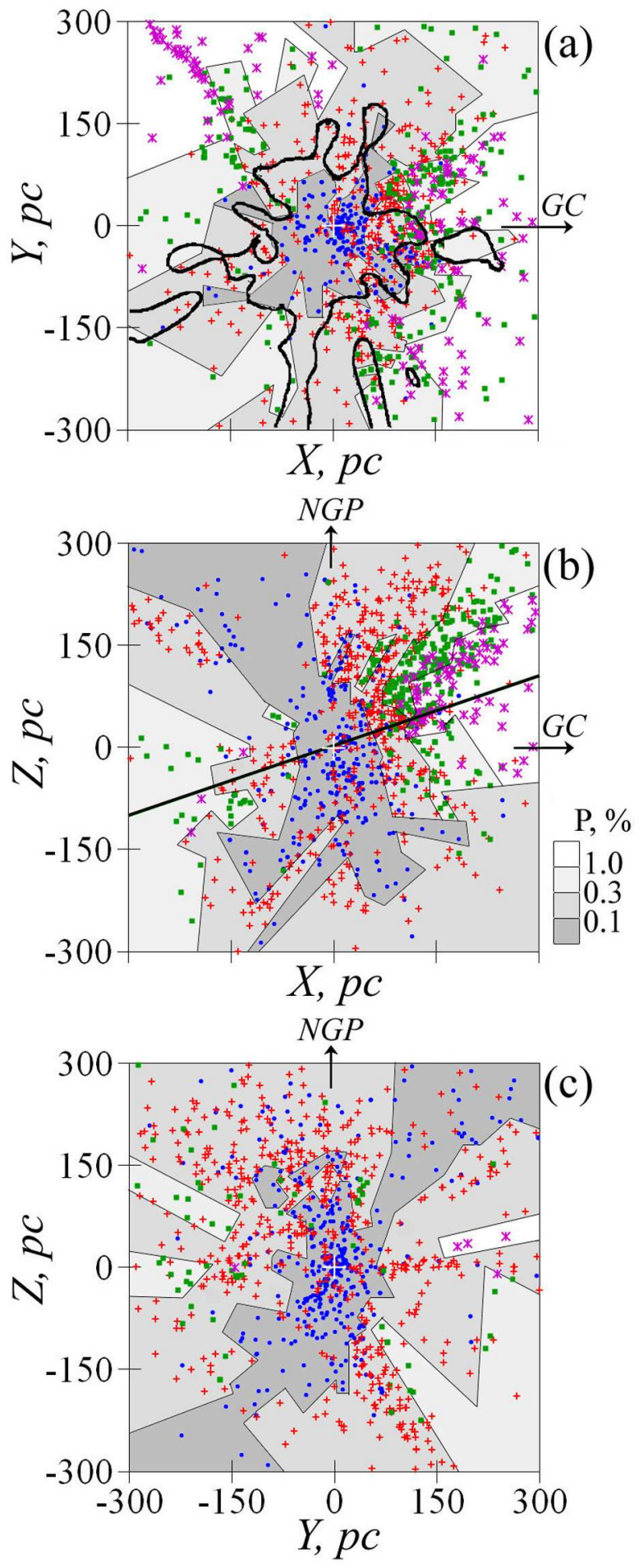

Figure 7. The distribution of the sample along the $X Y Z$ coordinates in the slices (a) $-60<Z<60 \mathrm{pc}$, (b) $-60<Y<60 \mathrm{pc}$, and (c) $-60<X<60$ pc. The stars with $P<0.1,0.1<P<0.33$, $0.33<P<1$ and $P>1$ per cent are shown by the blue dots, red crosses, green squares and purple snowflakes, respectively. The regions with these different levels of $P$ are shown by shades of grey. The black thick curve on the $X Y$ plot is the Na I volume density of $\log n_{\mathrm{Na}}=-9.1 \mathrm{~cm}^{-3}$ iso-contour from Welsh et al. (2010). The black thick line on the $X Z$ plot shows the orientation of the midplane of the Gould Belt. The directions to the Galactic centre (GC) and the North Galactic pole (NGP) are shown.
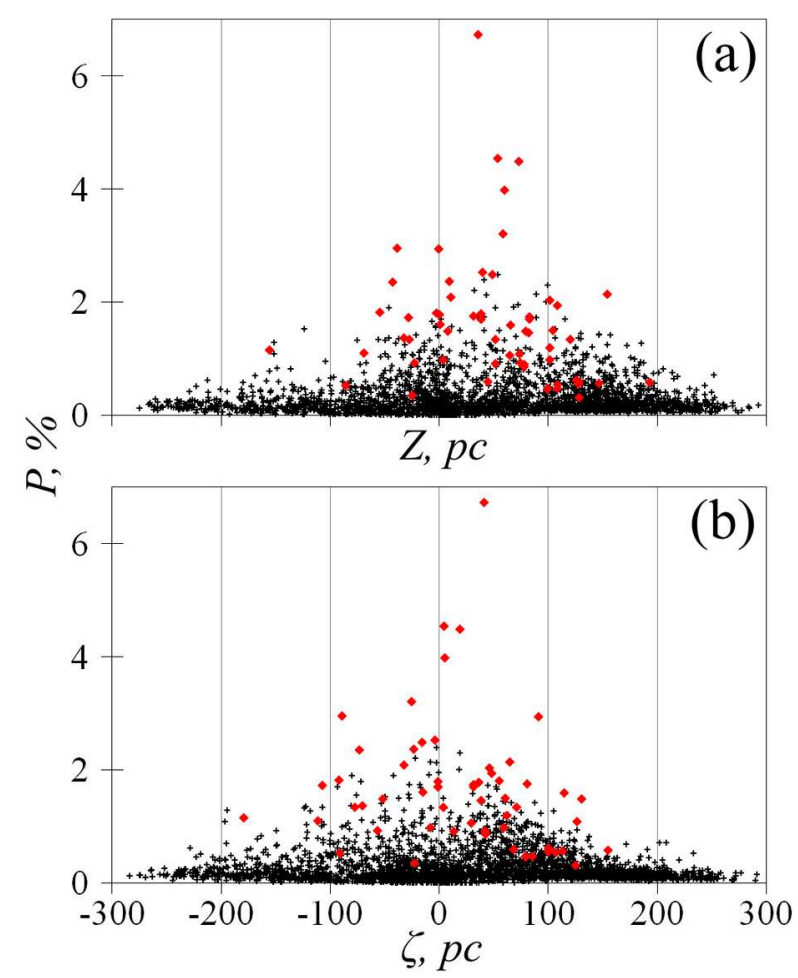

Figure 8. The distribution of the subsample limited by $R<300$ pc along (a) $Z$ and (b) $\zeta$. The 67 stars with $P / E(B-V)>9.3$ per cent per mag from all the reddening data sources are shown with the red diamonds. The error bars are not shown because they are mostly smaller than the depicted symbols. 


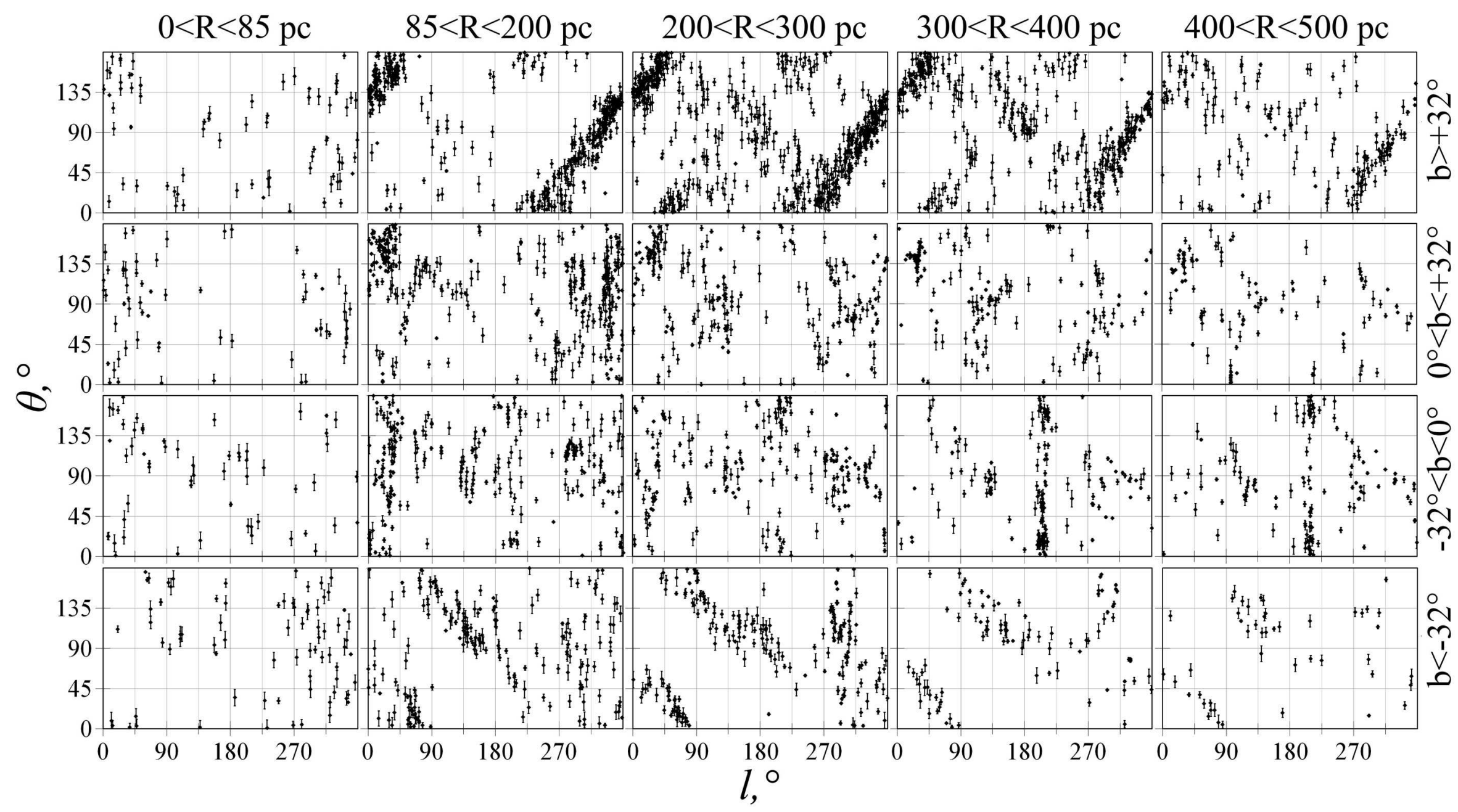

Figure 9. $\theta$ versus $l$ for the different latitude and distance segments. 


\subsection{Spatial variations of position angle}

We analysed the orientation of the polarisation vectors, i.e. their $\theta$, as a function of distance for four latitude zones: $b>+32^{\circ}, 0^{\circ}<b<+32^{\circ},-32^{\circ}<b<0^{\circ}$, and $b<-32^{\circ}$ in Fig. 9. A similar analysis with comparable results has been carried out by Stephens et al. (2011). The partitioning of the sky into these 4 zones is due to a rather different behaviour of $\theta$ in them. Also, this partitioning is partially explained by a non-uniform distribution of the sample (see Fig. 4) and by the domination of the data from Berdyugin, Piirola \& Teerikorpi (2014) at the high northern latitudes.

Almost no noticeable feature is seen inside the Local Bubble, i.e. in the leftmost plots of Fig. 9. This cannot be explained by a larger uncertainty of $\theta$ due to a lower $P$ inside the Bubble: the average $\sigma(\theta)$ is almost the same $-6^{\circ}$ and $5^{\circ}$ for $R<85$ and $R>85 \mathrm{pc}$, respectively. Such various $\theta$ for one $l$ suggest a chaotic magnetic field and/or chaotic orientation of dust grains and/or depolarisation of the polarised light by the radiation field of the Bubble. The latter reason would be in line with the drop of $P / R$ within the Bubble seen in Fig. 6 (b).

In contrast, the space outside the Bubble is full of noticeable features. In the northern high latitudes (the top plots) we see the same pattern for all $R$ : a regular bulk of stars draws a relation between $\theta$ and $l$ giving $\theta=0$ or $180^{\circ}$ at $l \approx 40^{\circ}$ and $l \approx 240^{\circ}$ (the last value drifts from $240^{\circ}$ to $270^{\circ}$ with increasing $R$, as discussed below). This relation points to a regular orientation of $\theta$ along a great loop with a radius of $(360-240+40) / 2=80^{\circ}$, i.e. nearly along a great circle. This loop is located near the Nothern Galactic pole at $l \approx 320^{\circ}$ where $\theta \approx 90^{\circ}$.

This feature has been known since the first all-sky interstellar polarisation maps (Mathewson \& Ford 1970; Leroy 1999). The highest part of this loop at $b \approx+80^{\circ}$ has long been known as the Markkanen's cloud of aligned dust (Markkanen 1979). Berdyugin \& Teerikorpi (2002) have shown that Markkanen's cloud extends to lower latitudes, apparently, being a part of a large elongated dust envelope. The Gaia DR2 parallaxes are quite accurate in order to recognise the large thickness of this envelope: Fig. 9 shows it at least for $85<R<400 \mathrm{pc}$. This agrees with a conclusion of Berdyugin et al. (2001) that the Markkanen's cloud starts at the distance of about $100 \mathrm{pc}$ and extends well beyond 300 pc. Moreover, this confirms the distance to the North Galactic Pole Rift, a well-defined H I feature at about 100 pc (Puspitarini \& Lallement 2012; Snowden et al. 2015), as a near part of the Markkanen's cloud.

Berdyugin, Piirola \& Teerikorpi (2014) have shown that the Markkanen's cloud exactly follows a large bright filament (hereafter the Markkanen's filament) of a higher dust far-IR emission from the emission/reddening map of Schlegel, Finkbeiner \& Davis (1998, hereafter SFD) (see figure 8 in Berdyugin, Piirola \& Teerikorpi). The Markkanen's filament is delineated by the largest black rectangle in the centre of the left plot of Fig. 10 reproducing the SFD map.

The next rectangle contains another filament in the $4^{\text {th }}$ quadrant in Fig. 10. This filament coincides with the wellknown North Polar Spur (NPS), one of the best known features in the radio continuum and diffuse soft X-ray background maps between $l \approx 25^{\circ}, b \approx+20^{\circ}$ and $l \approx 330^{\circ}$, $b \approx+75^{\circ}$ (Lallement et al. 2016). This NPS filament does not enter the $3^{r d}$ quadrant and has $\theta \approx 0$ or $180^{\circ}$ at $l \approx 270^{\circ}$. These properties allow us to find it as another region with aligned dust in Fig. 9. As mentioned above, the intersection of the bulk of stars with $\theta=0$ or $180^{\circ}$ in the top plots drifts from $l \approx 240^{\circ}$ to $270^{\circ}$ when $R$ increases from 85 to $500 \mathrm{pc}$. This means that the dominance of the Markkanen's filament in this bulk is changed by the dominance of the NPS filament when $R>300 \mathrm{pc}$. This agrees with a conclusion of Lallement et al. (2016), who have been ruled out the Spur's near side closer than $300 \mathrm{pc}$.

Another regular bulk of stars is seen in Fig. 9 at the high northern latitudes (the top plots) for $50^{\circ}<l<225^{\circ}$ and $200<R<500$ pc. It has an X-like shape in Fig. 9. This presents a loop of aligned dust delineated by the largest oval in the left plot of Fig. 10. The lowest latitude part of this loop is seen in the low northern latitude plots of Fig. 9 as a minor bulk of stars at $90^{\circ}<l<135^{\circ}, 200<R<400$ pc, and $\theta \approx 90^{\circ}$.

The last feature of aligned dust in the northern hemisphere is seen in Fig. 9 as an X-like bulk of stars at the low northern latitudes, the $4^{\text {th }}$ quadrant, $85<R<300$ pc. This depicts another loop seen in Fig.10 as a filament delineated by an oval in the $4^{\text {th }}$ quadrant. As seen from the Na I contour (the black curve) in Fig. 7 (a), this loop may be an interface between the Local and Loop I bubbles (Welsh et al. 2010). The Loop I Bubble is ' $80^{\circ}$ in radius and centred 120 pc away at $l=320^{\circ}, b=5^{\circ}$ for the neutral gas' (Frisch 2007). Leroy (1999); Reis et al. (2011); Santos, Corradi \& Reis (2011); Frisch et al. (2015); Cotton et al. (2017) discussed all the structures near this region as an interface between the Local and Loop I bubbles. In such a discussion one should take into account that Fig. 7 shows an elongation of the Local Bubble as a region with low polarisation both in this interface and in the place of the Loop I Bubble. However, Fig. 9 shows that the Local and Loop I bubbles are much smaller than their envelope of aligned dust.

This great envelope of the northern hemisphere contains the Markkanen's and NPS filaments and follows their orientation. Hence, this envelope is elongated between $l \approx 40^{\circ}$ and $250^{\circ}$ and, thus, nearly follows a wide extension of the Local Bubble at low latitudes of the $2^{\text {nd }}$ and $6^{\text {th }}$ octants, evident in Fig. 7 (a) and known as the Local interstellar tunnel, or the Great tunnel (Welsh 1991; Gontcharov 2012b). It consists of the Cygnus Cavities, Local Bubble, and CMa tunnel (see LVV, figure 1). This tunnel has many young stars and cloud complexes on its sides. Consequently, in fact, it defines the orientation of the Local spiral arm and its polarisation vectors.

As seen from Fig. 9 and 10, the great envelope enters the low latitudes where it fades in a strong emission/reddening/extinction. Yet, this envelope may re-appear as brightest filaments of the southern hemisphere. However, Fig. 4 shows that we have no enough data coverage of the largest southern filament, a curved one between the $1^{\text {st }}, 2^{\text {nd }}$ and $3^{r d}$ quadrants in the right plot of Fig. 10. Yet, two regular bulks of stars in Fig. 9 are the filaments of aligned dust inside the rectangles in the right plot of Fig. 10. The filaments in the larger rectangle in the $4^{\text {th }}$ quadrant are represented in Fig. 9 by the bulk of stars with $\theta \approx 90^{\circ}$ at the low southern latitudes, $85<R<500$ pc. In fact, Fig. 9 shows similar regular bulks in the $2^{d}$ and $4^{\text {th }}$ quadrants at the low 


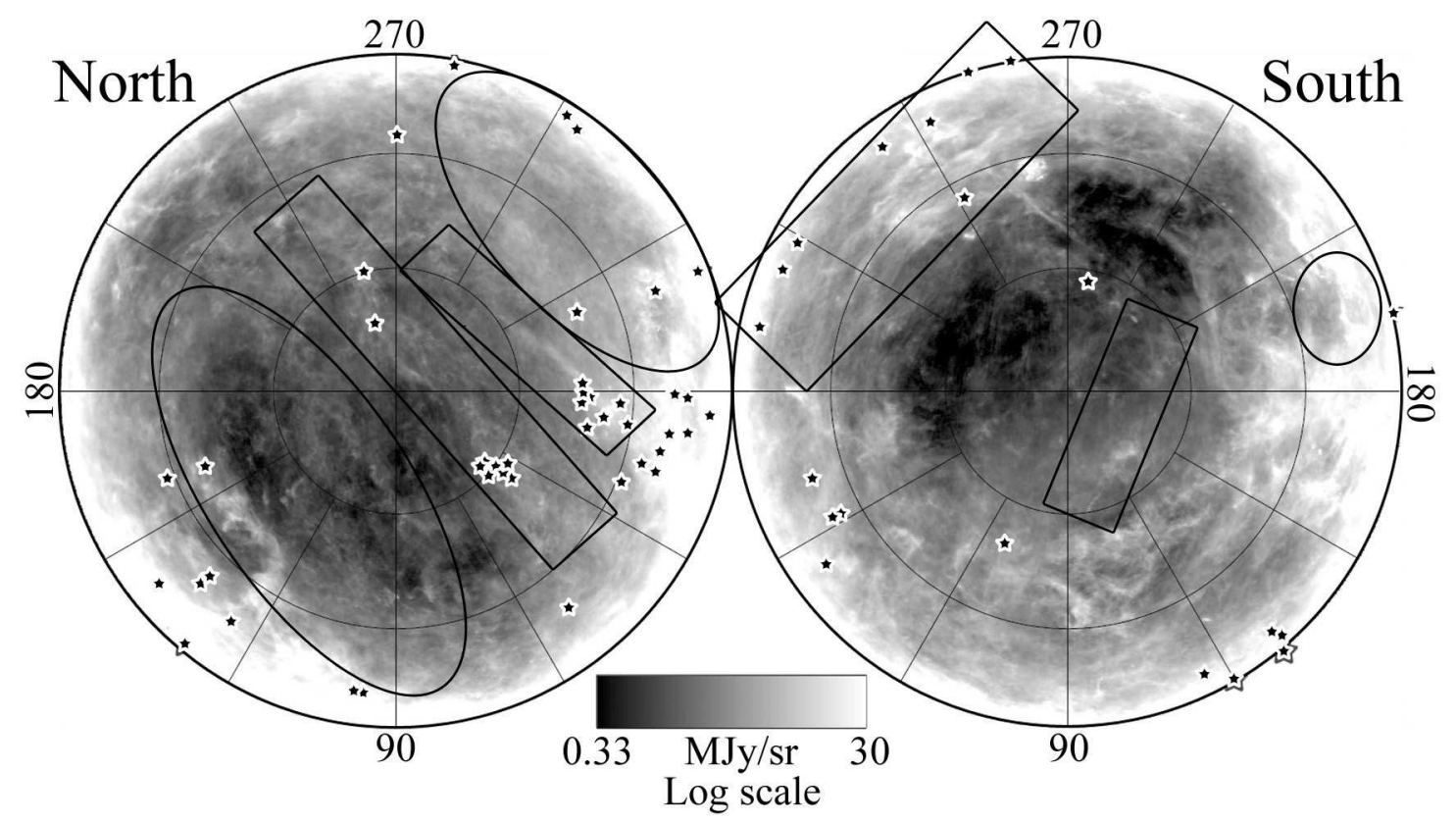

Figure 10. The 67 stars with $P / E(B-V)>9.3$ per cent per mag from all the reddening data sources superimposed over the SFD map. Some high latitude areas of higher emission and reddening are shown by rectangles, while some polarisation loops are shown by ovals.

northern and southern latitudes. This suggests a prolongation of the discussed northern loops (the two ovals in the left plot of Fig. 10) in the South.

The smaller rectangle in the $1^{\text {st }}, 2^{\text {nd }}$ and $3^{r d}$ quadrants in Fig. 10 is represented in Fig. 9 by a pronounced bulk of stars at the high southern latitudes, $85<R<400$ pc. By their orientation and $\theta$ these southern filaments may be a prolongation of the great northern envelope. More data are needed to make more robust conclusions on this envelope.

The last feature in the South is a compact loop in Orion delineated by the oval in the right plot of Fig. 10. It is seen in Fig. 9 as a sharp vertical bulk of stars at $b \approx 208^{\circ}$ in the low southern latitudes within $300<R<500$ pc. It has a chaotic $\theta$.

We note 67 stars with $P / E(B-V)>9.3$ per cent per mag shown in Fig. 10 by the star symbols. Most of them appear at (i.e. before, in or behind) the middle and high latitude filaments. We discuss these stars in Section 3.3.

\section{POLARISATION VERSUS REDDENING}

\subsection{Reddening data}

Reddening data sources, in contrast to polarisation data sources, are inhomogeneous in their origin and methods. Their large systematic errors do not allow one to use them in many cases. For example, only five of the thirteen selected publications on polarisation give a comparison between the polarisation degree and the reddening. Heiles (2000) considered $E(B-V)$, but with very low precision of 0.1 mag in almost all cases. Santos, Corradi \& Reis (2011) found $E(b-y)$, but only for 5 per cent of their stars with polarisation measurements. Alves \& Franco (2006), Poidevin \& Bastien (2006), and Andersson \& Potter (2007) used reddening and extinction estimates, but only in some small areas of the sky.
Table 5. The linear correlation coefficients of $P$ (per cent) and $E(B-V)(\mathrm{mag})$ from the different reddening data sources and from Gaia DR2 inside $(P<0.1$ per cent, the top right half of the Table) and outside $(P>0.1$ per cent, the bottom left half of the Table) the Bubble.

\begin{tabular}{cccccccr}
\hline \multicolumn{7}{c}{ Inside } \\
\hline Outside & $\boldsymbol{P}$ & G17 & G12 & AGG & DCL & LVV & DR2 \\
\hline $\boldsymbol{P}$ & & 0.31 & 0.38 & 0.24 & 0.27 & 0.35 & -0.06 \\
G17 & 0.59 & & 0.64 & 0.48 & 0.46 & 0.59 & -0.06 \\
G12 & 0.50 & 0.76 & & 0.67 & 0.57 & 0.71 & -0.01 \\
AGG & 0.56 & 0.73 & 0.69 & & 0.38 & 0.46 & 0.00 \\
DCL & 0.08 & 0.16 & 0.20 & 0.04 & & 0.49 & 0.01 \\
LVV & 0.66 & 0.80 & 0.69 & 0.72 & 0.02 & & 0.04 \\
DR2 & 0.06 & 0.04 & 0.03 & 0.05 & 0.00 & 0.07 & \\
\hline
\end{tabular}

Recently, reddening estimates were obtained for millions lines of sight for the whole sky by use of farIR dust emission and stellar multi-colour photometry. Gontcharov \& Mosenkov (2017a,b, 2018) have tested the random and systematic accuracy of various reddening data sources through their ability to fit the precise photometry and parallaxes of the stars from the Gaia DR1 Tycho-Gaia Astrometric Solution (Michalik, Lindegren \& Hobbs 2015) to some theoretical estimates in the HR diagram. The theoretical estimates are based on the PARSEC (Bressan et al. 2012) and MIST (Dotter 2016) theoretical isochrones, and the TRILEGAL model of the Galaxy (Girardi et al. 2005). Gontcharov \& Mosenkov (2017a,b, 2018) concluded that the following 3D data sources (maps and models) are the most reliable within several hundred parsecs from the Sun: Arenou, Grenon \& Gomez (1992, hereafter AGG), 
Table 6. The 67 stars with $P / E(B-V)>9.3$ per cent per mag by the reddening estimates from all the data sources: the Gaia DR2 source_id or Hipparcos number, $G$ magnitude, distance (parsecs), Galactic coordinates (degrees), $P$ and its $\sigma$ (per cents), $\theta$ and its $\sigma$ (degrees), $R_{\mathrm{V}}$, and $E(B-V)$ from G17, G12, AGG, DCL, and LVV. The complete table is available online.

\begin{tabular}{|c|c|c|c|c|c|c|c|c|c|c|c|c|c|c|}
\hline DR2 Source_id or HIP & $G$ & $R$ & $l$ & $b$ & $P$ & $\sigma(P)$ & $\theta$ & $\sigma(\theta)$ & $R_{\mathrm{V}}$ & G17 & G12 & AGG & DCL & LVV \\
\hline 1037320780504503040 & 7.95 & 429 & 159.6896 & +37.8749 & 0.975 & 0.054 & 95 & 2 & 3.22 & 0.097 & 0.075 & 0.013 & 0.092 & 0.027 \\
\hline 1264203385691704448 & 8.96 & 150 & 36.7309 & +58.1949 & 0.560 & 0.028 & 172 & 1 & 3.59 & 0.040 & 0.056 & 0.015 & 0.028 & 0.032 \\
\hline 1264661680178370432 & 5.42 & 149 & 37.0262 & +59.2407 & 0.598 & 0.120 & 156 & 6 & 3.59 & 0.037 & 0.055 & 0.015 & 0.035 & 0.029 \\
\hline 1264797229346052864 & 4.15 & 114 & 36.2511 & +60.6605 & 0.478 & 0.029 & 150 & 2 & 3.59 & 0.005 & 0.050 & 0.027 & 0.026 & 0.020 \\
\hline 1265048261594583552 & 8.34 & 127 & 37.8240 & +58.8987 & 0.484 & 0.010 & 161 & 1 & 3.59 & 0.015 & 0.052 & 0.015 & 0.029 & 0.026 \\
\hline$\ldots$ & $\ldots$ & $\ldots$ & $\ldots$ & $\ldots$ & $\ldots$ & $\ldots$ & $\ldots$ & $\ldots$ & $\ldots$ & $\ldots$ & $\ldots$ & $\ldots$ & $\ldots$ & $\ldots$ \\
\hline
\end{tabular}

Drimmel, Cabrera-Lavers \& López-Corredoira (2003, hereafter DCL), ${ }^{1}$ Gontcharov (2009, 2012b, hereafter G12), and G17.

In this study we use these data sources together with a new 3D map by LVV, which may be especially useful for the space under consideration. LVV provides the reddening only within $|Z|<300$ pc. Yet, since the Galactic dust layer lies mostly within $|Z|<300 \mathrm{pc}$, we use the reddening at $Z= \pm 300 \mathrm{pc}$ for $Z> \pm 300 \mathrm{pc}$ along the same line of sight.

With the median $|Z|=122 \mathrm{pc}$, most stars of our sample are located inside the inhomogeneous Galactic dust layer. The authors of the selected reddening data sources have made some efforts to take it into account (Gontcharov \& Mosenkov 2017b). However, their median $E(B-V)$ for our sample are very diverse: $0.087,0.075,0.048$, 0.038 , and $0.027 \mathrm{mag}$ for G17, G12, AGG, DCL, and LVV, respectively. The difference between these values is higher than the formal precision of these data sources. Therefore, the following mutual analysis of $P, E(B-V)$, and $P / E(B-V)$ may help to select the most reliable reddening estimates.

Reddening estimates from many other data sources are not applicable to stars inside the dust layer within $500 \mathrm{pc}$ from the Sun, as pointed out by Gontcharov \& Mosenkov (2017b, 2018). An example is the 2D (to infinity) reddening maps by SFD and Meisner \& Finkbeiner (2015), based on the observations of the dust emission in far-IR by $C O B E$, $I R A S$, and Planck. The reduction of their reddening from infinity to a point inside the dust layer gives a reliable result only by use of either a model of the local dust spatial distribution (as realised in DCL), or some photometry of local stars. Note that $3 \mathrm{D}$ reddening maps based on photometry of distant stars are not reliable in the nearby space. An example is the map of Green et al. (2018), which provides a reliable reddening only for a minority of our stars.

Gaia DR2 also provides the reddening and extinction, but only for 68 per cent of our stars. Moreover, these reddenings and their very large scatter for close lines of sight seem to be unreliable. For example, given the extinction law of Cardelli, Clayton \& Mathis (1989), Gaia DR2 gives for 658 stars at $|b|>50^{\circ}$ the median $E(B-V)=0.131$ mag with its standard deviation 0.128 mag due to a natural diversity of the medium. These values strongly disagree with the values $0.047 / 0.017,0.041 / 0.013,0.022 / 0.012,0.019 / 0.009$, and

1 The DCL estimates are calculated by use of the code of Bovy et al. (2016), https://github.com/jobovy/mwdust

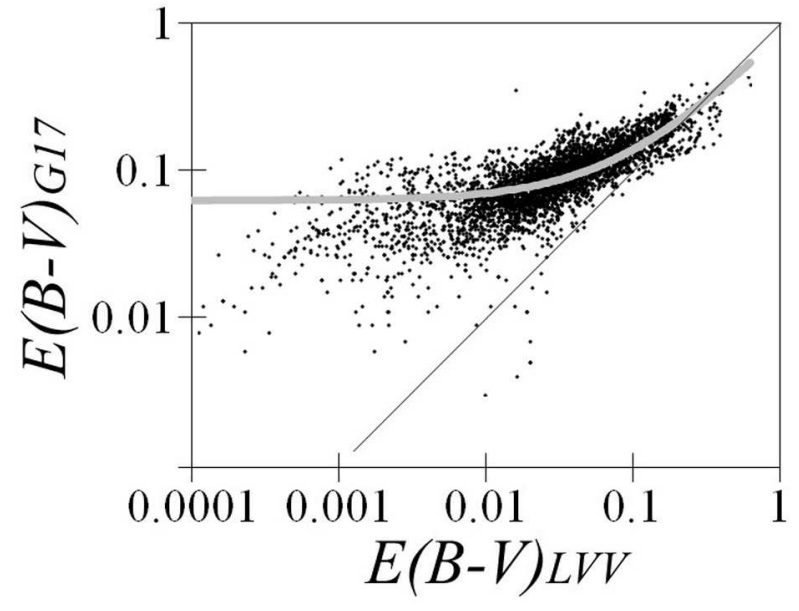

Figure 11. $E(B-V)$ from LVV versus G17 for our sample. The linear trend is shown by the grey thick line, while the one-to-one relation - by the black thin line.

0.012/0.008 mag from G17, G12, AGG, DCL, and LVV, respectively, for the same stars. Therefore, we do not use the Gaia DR2 reddening estimates.

However, we consider the reddening estimates from the Gaia DR2, recalculated into $E(B-V)$ by use of the extinction law of Cardelli, Clayton \& Mathis (1989) with $R_{\mathrm{V}}=3.1$, among other estimates of $E(B-V)$ and the estimates of $P$, to calculate their linear correlation coefficients. They are presented in Table 5. The reddenings from the Gaia DR2 show a very weak correlation with both $P$ and the remaining reddening estimates both in- and outside the Bubble. The correlations between $P$, G17, G12, AGG, and LVV are fairly tight, especially, outside the Bubble. Given a rather significant differences between G17, G12, AGG, and LVV, their high correlation coefficients mean that their reddening estimates can be basically described as some linear trends of each other. Indeed, Fig. 11 shows $E(B-V)$ estimates from LVV versus G17. The linear trend

$E(B-V)_{G 17}=0.06+0.76 E(B-V)_{L V V}$,

shown by the thick grey curve, reveals that LVV and G17 differ mostly by the zero-point of 0.06 mag, most significant at high latitudes. Besides this, Fig. 11 shows a noticeable bulk of stars with $E(B-V)_{G 17}<0.04$ and $E(B-V)_{L V V}<$ 0.001 . These are stars within $40 \mathrm{pc}$. Their $E(B-V)_{L V V}$ seem to be well below the level which can actually be measured by 
photometry. Yet, a low accuracy of both $P$ and $E(B-V)$ does not allow us to make any conclusion on this nearby space.

\subsection{Dependences on $R$}

Fig. 12 shows $P / E(B-V)$ versus $R$ for (a) G17, (b) G12, (c) AGG, (d) DCL, and (e) LVV. It is seen that there is a slightly higher scatter of $P / E(B-V)$ for all the reddening data sources within nearly $R<100 \mathrm{pc}$ due to lower values and, consequently, higher fractional errors of both $P$ and $E(B-V)$ (see a discussion in Frisch et al. (2015) regarding their figure 2). Yet, Fig. 12 shows a clear drop of $P / E(B-V)$ within nearly $R<100$ pc by use of the data from G17, G12, and AGG. Such a drop is not so evident for DCL, while LVV clearly shows a monotonic decrease of $P / E(B-V)$ with $R$ and a little depression of $P / E(B-V)$ only within $R<50 \mathrm{pc}$, in contrast to the other reddening data sources. Comparing the spatial variations of $P$ and $P / R$ from Fig. 6 , on the one hand, with the ones of $P / E(B-V)$ from Fig. 12, on the other hand, we can conclude that LVV shows a drop of $E(B-V) / R$ inw.r.t. outside the Bubble similar to the one of $P / R$, whereas the remaining reddening data sources show no considerable drop of $E(B-V) / R$ at all.

Thus, choosing between the reddening estimates from LVV or other data sources, we encounter a key problem for understanding the nature of the local interstellar medium, whether $E(B-V)$ follows $P$ in its spatial variations. We note that $E(B-V)$ and $P$ have different nature. The latter is a percentage of the aligned dust, it is limited between 0 and 100 per cent, and it can either increase or decrease with $R$. In contrast, $E(B-V)$ has no upper limit and cannot decrease with $R$. Therefore, $E(B-V)$ and $P$ do not necessarily follow each other.

For 543 stars with $P=0.10 \pm 0.02$ per cent, i.e. at the boundary of the Bubble the median $E(B-V)$ are 0.067, 0.056, 0.029, 0.025, and 0.014 mag from G17, G12, AGG, DCL, and LVV, respectively. The diversity of these estimates is similar to the diversity of the Bubble's boundary estimates mentioned in Section 1. Therefore, given the nearly constant value of $P$ at the boundary of the Bubble, we expect such a large diversity of the estimates of $P / E(B-V)$ to be with the median 1.5, 1.8, 3.4, 4.0, and 7.1 for G17, G12, AGG, DCL, and LVV, respectively. We discuss it in Section 3.3.

Our results on the drops of $P / R, P / E(B-V)$ and, hence, $E(B-V) / R$ inside the Bubble are compatible with the results of Welsh et al. (2010). They have studied the equivalent width, as well as the column and volume density of $\mathrm{NaI}$ and CaII absorption lines towards 1857 stars. By use of $\mathrm{NaI}$ and $\mathrm{Ca}$ II they have determined the 3D spatial distribution of neutral and partly ionised interstellar gas density, respectively. Dust exists in both neutral and ionised regions. But it is aligned to provide a considerable polarisation in the neutral regions only. The Bubble is a space with many warm and partially ionised low density Ca II-bearing cloudlets (Welsh et al. 2010). Hence, the Ca II marker should better correspond to the dust volume density and $E(B-V) / R$, whereas the Na I marker - to the polarisation degree. This agrees with the conclusion of Bailey et al. (2010) that 'the dust responsible for the polarization is not located in the clouds responsible for the CaII absorption.' Welsh et al. (2010) have revealed different drops of $\mathrm{Na}$ I and Ca II volume densities inside the Bubble (see their figures 9 and 10).

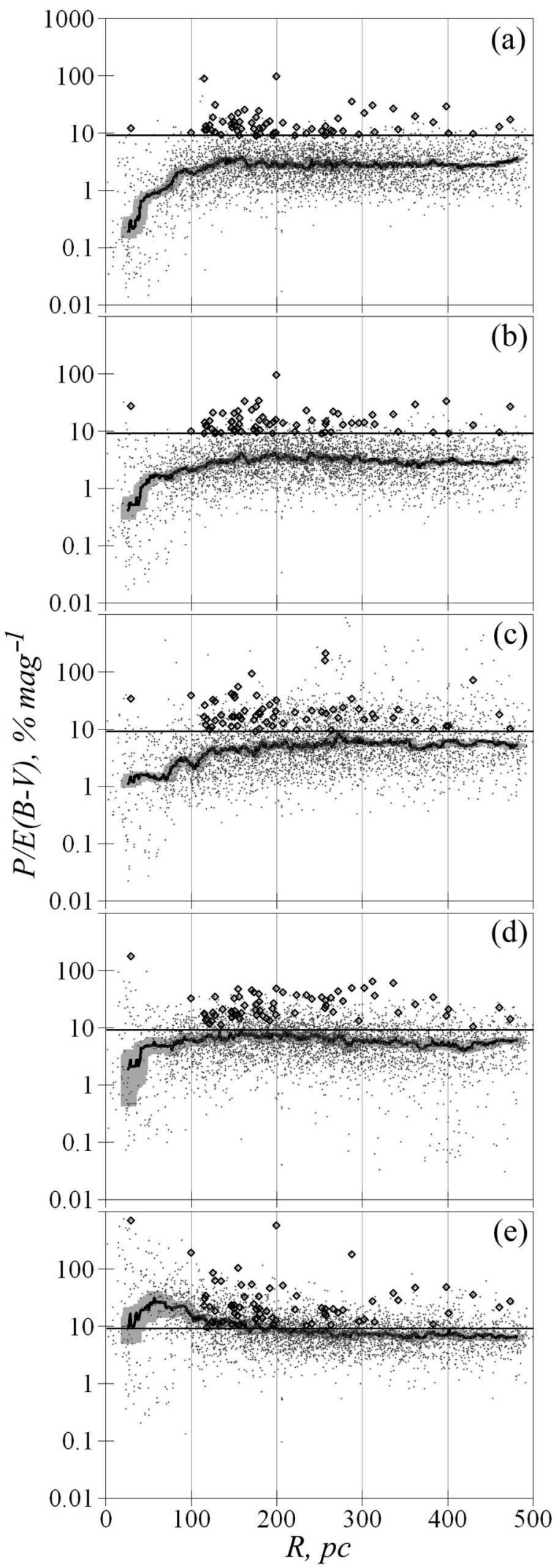

Figure 12. $P / E(B-V)$ (per cent per mag) versus $R$ (parsecs) for the (a) G17, (b) G12, (c) AGG, (d) DCL, and (e) LVV $E(B-V)$ estimates. All the curves are the moving medians over 101 points. The 67 stars with $P / E(B-V)>9.3$ per cent per mag from all the reddening data sources are shown with the open diamonds. 


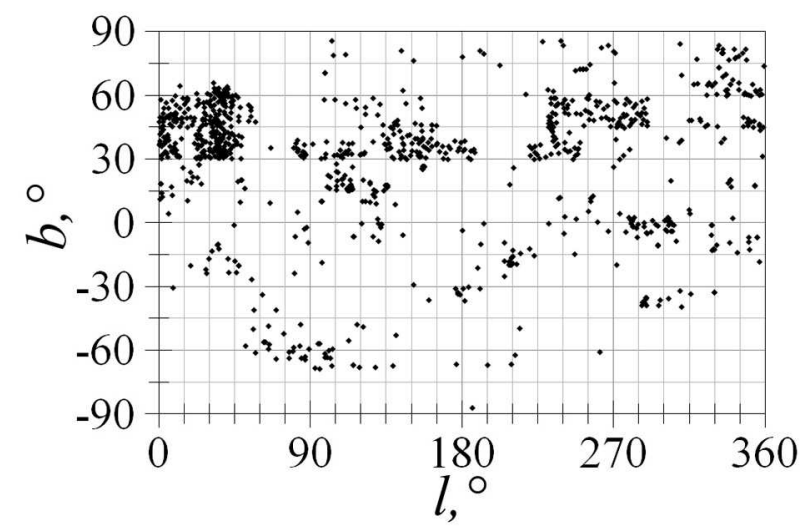

Figure 13. The distribution of stars with $P / E(B-V)>9.3$ per cent per mag by use of AGG in the sky.

We have re-analysed their data by use of the Gaia DR2 parallaxes. Taken $\varpi>14$ and $3<\varpi<7$ mas for the space in- and outside the Bubble, respectively, we have found the drops by 6.2 and 1.5 times for NaI and Ca II volume densities, respectively. The former value agrees with the above mentioned drop of $P / R$, whereas the latter value agrees with the negligible drop of $E(B-V) / R$ by use of all the reddening data sources, except LVV. Hence, the drop of $E(B-V) / R$ from LVV by an order of magnitude makes them the outlier and leads to too many stars with too high $P / E(B-V)$. Moreover, very high $P / E(B-V)$ in the Bubble from LVV strongly contradicts to the chaotic $\theta$ there, which is evident from Fig. 9. Therefore, we are inclined to conclude that LVV systematically underestimates $E(B-V)$ inside the Bubble.

It seems that the Bubble differs from the surrounding space by slightly lower volume density of dust (and $E(B-V) / R$ as its proxy) and about 6 times lower volume density of neutral gas (and $P / R$ as its proxy). Yet, an overall gasto-dust ratio in the Bubble can be maintained due to a several times higher volume density of ionised gas. Frisch et al. (2015) gave an example of a region inside the Bubble (BICEP2 field) where the stellar reddening suggests the dust column density about 6.7 times higher than the neutral gas column density derived from the polarisation data.

Thus, a relation between the polarisation degree and reddening for any large volume of space with the inhomogeneous medium inside, such as the equation (1) from Fosalba et al. (2002), has no sense. Instead, one should consider such relations separately for every space region with homogeneous medium.

\subsection{Serkowski's limit}

The black horizontal lines in Fig. 12 show the Serkowski's limit $P / E(B-V)=9.3$ per cent per mag. It is seen that the reddening data sources give different number of cases with $P / E(B-V)>9.3$ per cent per mag: 148 (3.8 per cent), 208 (5.4 per cent), 929 (24 per cent), 1033 (27 per cent), and 1660 stars (43 per cent) for G17, G12, AGG, DCL, and LVV, respectively. Even among 3520 stars with $R>100 \mathrm{pc}$, where the scatter of $P / E(B-V)$ is certainly lower, these numbers are 138 (3.9 per cent), 198 (5.6 per cent), 896 (25 per cent), 945 (27 per cent) and 1405 (40 per cent), respectively. These percentages can be compared with 4 per cent of such stars in
Planck Collaboration et al. (2015) and few per cents in the other studies. By their very large percentage, AGG, DCL, and LVV, in fact, reject the Serkowski's limit. Consequently, we must conclude that either Serkowski's limit is not valid at all, or AGG, DCL, and LVV considerably underestimate reddening within $500 \mathrm{pc}$, or $P / E(B-V)$ are strongly biased due to the selection effect in favour of stars with higher $P$. The latter is quite unprobable, as follows from the agreed variations of $P$ in Fig 7 .

The stars with $P / E(B-V)>9.3$ per cent per mag have non-uniform distribution on the sky and along $R$. For example, such stars selected by use of AGG follow a coordinate partitioning during the creation of the AGG model. Namely, one can see in Fig. 13 that the majority of these stars drop exactly to a few cells of this partitioning (see the Appendix of AGG): $15<b<30$ with $100<l<140 ; 30<b<45$ with $0<l<50 ; 30<b<45$ with $80<l<190 ; 30<b<45$ with $220<l<250 ; 45<b<60$ with $0<l<60 ; 45<b<60$ with $110<l<170 ; 45<b<60$ with $230<l<290$; and $b>60$ with $330<l<360$. Most of these stars have $R>250$. Apparently, the main reason of $P / E(B-V)>9.3$ per cent per mag in this case is an underestimation of $E(B-V)$ in these cells at rather large distances. In turn, it is due to a poor sample of the distant stars used in the creation of AGG in these cells. DCL and LVV show a higher percentage of the stars with $P / E(B-V)>9.3$ per cent per mag within $R<220$ pc and $R<150 \mathrm{pc}$, respectively, due to their very low reddening estimates near the Sun. However, DCL admits this reddening as underestimated due to 'the spatial resolution of the [DCL] dust model does not allow a detailed description of dust in the vicinity of the Sun' (DCL), while LVV insists on such a very low reddening in uncompromising contrast to the Serkowski's limit.

Any case of $P / E(B-V)>9.3$ per cent per mag for a star by use of the only reddening data source can be explained by an underestimation of the reddening for this star by this data source. Yet, we found 67 stars with $P / E(B-V)>9.3$ per cent per mag based on the reddening estimates from all five data sources. Our analysis of the SIMBAD data of these stars has revealed no indication of their intrinsic polarisation. These stars are presented in Table 6 and shown in Fig. 3, 4, 6, 8, 10, and 12 as the diamonds or stars. It is seen from these figures that these stars represent all stellar classes and tend to lie in a few sky areas and within the limited range $98<R<314$ pc ( 57 of 67 stars). Fig. 10 shows these stars superimposed over the SFD map. It is seen that they tend to concentrate near the Galactic centre, the Markkanen's cloud, the North Polar Spur, and some filament dust clouds. Probably, $P / E(B-V)>9.3$ per cent per mag for these stars is due to an underestimation of $E(B-V)$ in these regions.

On the other hand, the correlation coefficient between $R_{\mathrm{V}}$ from Gontcharov (2012a) and $\log (P)$ for these stars is -0.65 (compared with a negligible correlation for the whole sample). For most of these 67 stars with $P / E(B-V)>9.3$ per cent per mag we can obtain $P / A_{\mathrm{V}}<3$ (another version of the Serkowski's limit) by an acceptable deviation of $R_{\mathrm{V}}$ from the value 3.1. Consequently, the too high $P / E(B-V)$ of these stars may be explained by a deviation of the extinction law near them from the standard one.

Voshchinnikov, Il'in \& Das (2016) have explained the existence of stars with $P / E(B-V)>9.3$ per cent per 
mag within their theoretical dust models considering oblate grains with axial ratio $>3$. Such grains do not need to be perfectly aligned with the magnetic field to account for values above the Serkowski's limit. Voshchinnikov, Il'in \& Das even found $P / E(B-V)=9.5 \pm 2.5$ and $12.6 \pm 3.0$ per cent per mag for the Chamaeleon I and Musca clouds, respectively, by use of the data from Andersson \& Potter (2007). However, Voshchinnikov, Il'in \& Das found such an overcoming of the Serkowski's limit only for 2 out of 11 clouds within 500 pc, while LVV suspect this to be for almost a half of the stars. Therefore, this explanation seems to be valid only in some regions of the space. As seen from Fig. 4 and 6, several our stars are in such a special region of Chamaeleon I and Musca clouds, i.e. at $R=196 \mathrm{pc}, l=297^{\circ}, b=-15^{\circ}$ and $R=171 \mathrm{pc}, l=300^{\circ}, b=-9^{\circ}$, respectively. Moreover, most or even all the 67 stars with $P / E(B-V)>9.3$ per cent per mag from all the reddening data sources may be in such regions. Anyway, the current data are not enough to exclude these stars from our study, especially, since their influence on our results is negligible.

\subsection{Systematics of LVV}

Inexplicably large $P / E(B-V)$ from LVV leads us to a discussion on their possible systematic errors (naturally, not excluding some systematic errors in the reddening data from the other sources).

The stellar sample used to create LVV is far from being complete. For example, it contains less than 20,000 stars within $40<R<100 \mathrm{pc}$, as compared to a complete sample of about 50,000 turn-off stars used to create G17 in the same space. Moreover, 'There is a limitation in the brightness of the target stars, and the subsequent lack of strongly reddened stars results... There is for the same reasons a bias towards low opacities...' (LVV).

LVV determined the reddening as $E(b-y)=(b-y)-$ $(b-y)_{0}$, where the intrinsic colour $(b-y)_{0}$ was calculated according to the spectral type of the star. Intrinsic colours can be overestimated due to the well-known difficulties in the selection of unreddened stars. LVV stated the uncertainty $\sigma(E(B-V)) \approx 0.02 \mathrm{mag}$ for their data, keeping in mind an offset of $0.02 \mathrm{mag}$ found (and corrected) at the origin of the linear relationship between the Strömgren and Geneva photometric data. However, such an additional systematic offset of $\Delta E(B-V)=0.02 \mathrm{mag}$, if it is still hidden in the LVV data, would explain a major part of its deviation from the rest data sources. Such an offset would reconcile $E(B-$ $V)_{G 17}<0.04$ and $E(B-V)_{L V V}<0.001$ of the stars within 40 pc, shown in Fig 11 and discussed in Section 3.1.

At high latitudes, behind the dust layer, an offset of $\Delta E(B-V)=0.06 \mathrm{mag}$ would be more appropriate, as evident from Fig. 11, equation (4) and the discussion in Section 3.1. This would be in line with the conclusion of Gontcharov \& Mosenkov (2018) that the median $0.04<$ $E(B-V)<0.06$ through the dust half-layer at $|b|>50^{\circ}$ gives the best agreement of the distribution of the Gaia DR1 stars in the HR diagram with the theoretical predictions from PARSEC, MIST, and TRILEGAL, mentioned in Section 3.1. LVV noted 'The solution is based not only on the color excess data but also on prior knowledge of the opacity distribution... These two information sources complement each other: where the constraints from the data are insufficient, the inversion restores the prior density...'. At high latitudes, behind the dust layer, the approach of LVV sets the prior to $E(B-V)=0$, then the sparse distribution of their high latitude stars (they even had to limit their map by $|Z|<300$ pc) keeps this zero prior for the final map. This may be an explanation of a systematic underestimation of the reddening by LVV.

\subsection{Dependences on colour and Galactic coordinates}

In the following figures we show $P, E(B-V)$, and $P / E(B-V)$ versus the dereddened colour $\left(G_{B P}-G_{R P}\right)_{0}, b$ and $l$ only outside the Bubble, since the results inside are less accurate.

Fig. 14 shows (a) $R$, (b) $P$, (c) $E(B-V)$ and (d) $P / E(B-V)$ versus the dereddened colour $\left(G_{B P}-G_{R P}\right)_{0}$ for stars with $R>100$, based on the reddening estimates from G17, G12, AGG, DCL, and LVV.

Fig. 14 shows some large variations of $R, P$, and $E(B-V)$ with colour. However, these variations almost completely compensate each other, resulting in a nearly constant $P / E(B-V)$ as a function of colour. Particularly, the wellknown higher $P$ of $\mathrm{OB}$ and $\mathrm{M}$ stars, seen in Fig. 14 (b), is explained by their higher $R$ and compensated by their higher $E(B-V)$. This is true for all the reddening data sources, despite very different estimates of $E(B-V)$ and $P / E(B-V)$ for the same colour by various data sources. Moreover, DCL even shows a significant decrease of $P / E(B-V)$ for $\mathrm{OB}$ stars w.r.t. other classes. Yet, this is due to their very high $E(B-V)$, which can be erroneous.

Fig. 15 shows (a) $P$, (b) $E(B-V)$, and (c) $P / E(B-$ $V)$ versus $b$ for the stars with $R>100 \mathrm{pc}$, based on the reddening estimates from G17, G12, AGG, DCL, and LVV. Both $P$ and $E(B-V)$ show a rise within $|b|<20^{\circ}$ due to the influence of the Gould Belt, as a dust container. There is also a sharp peak near $b \approx-19^{\circ}$ due to a large number of stars near the directions to the Orion clouds $\left(l \approx 208^{\circ}\right)$, where the Belt has a large deviation from the Galactic equator.

The orange dashed curve shows an approximating function obtained by Fosalba et al. (2002). A rather poor agreement on this curve with our data is due to the fact that Fosalba et al. (2002) considered all the stars within several kiloparsecs from the Sun.

As for the dependence on colour in Fig. 14 (d), it is seen from Fig. 15 (c) that the large variations of $P$ and $E(B-V)$ with $b$ almost completely compensate each other, resulting in a nearly constant $P / E(B-V)$, albeit at some different levels for different reddening data sources. Some residual variations of $P / E(B-V)$ on $b$ can be explained: for AGG - by very low accuracy at high latitudes, which is seen in the very wide error belt; for DCL - by a non-uniform spatial distribution of our sample. LVV is the only reddening data source showing a significant increase of $P / E(B-V)$ with $|b|$. Since it has no natural explanation, it should be considered as a confirmation of the above mentioned systematic underestimation of $E(B-V)$ at high latitudes by LVV.

For the dependences on colour and $b$, we use the limit $R>100$ pc. We do not use the limit $P>0.1$ per cent instead, i.e. in order to select the space exactly outside the Bubble, because it excludes the high latitude 'chimneys' with low $P$. This would result in a poor statistics on $b$ and a bias in the statistics on some stellar classes due to their different 

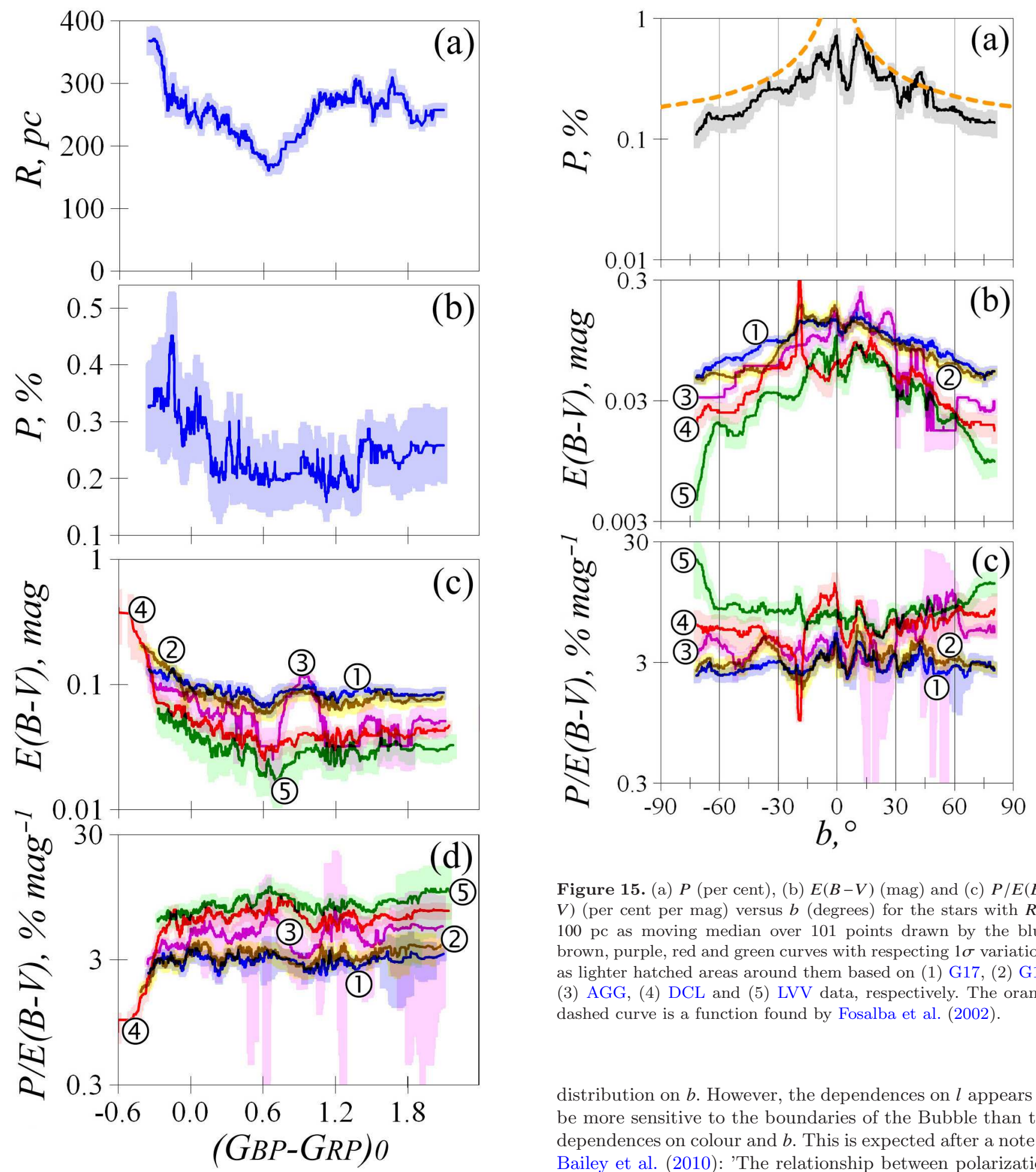

Figure 15. (a) $P$ (per cent), (b) $E(B-V)$ (mag) and (c) $P / E(B-$ $V$ ) (per cent per mag) versus $b$ (degrees) for the stars with $R>$ $100 \mathrm{pc}$ as moving median over 101 points drawn by the blue, brown, purple, red and green curves with respecting $1 \sigma$ variations as lighter hatched areas around them based on (1) G17, (2) G12, (3) AGG, (4) DCL and (5) LVV data, respectively. The orange dashed curve is a function found by Fosalba et al. (2002).

Figure 14. (a) $R$ (parsecs), (b) $P$ (per cent), (c) $E(B-V)$ (mag) and (d) $P / E(B-V)$ (per cent per mag) versus the dereddened colour $\left(G_{B P}-G_{R P}\right)_{0}(\mathrm{mag})$ for the stars with $R>100 \mathrm{pc}$ as moving median over 101 points drawn by the blue, brown, purple, red and green curves with respecting $1 \sigma$ variations as lighter hatched areas around them based on the (1) G17, (2) G12, (3) AGG, (4) DCL and (5) LVV data, respectively. Only the results from G17 are shown in the plots (a) and (b) due to a similarity of the results from all the reddening data sources.

distribution on $b$. However, the dependences on $l$ appears to be more sensitive to the boundaries of the Bubble than the dependences on colour and $b$. This is expected after a note of Bailey et al. (2010): 'The relationship between polarization and angle to the line of sight should lead to a polarization that depends on galactic longitude'. Therefore, Fig. 16 shows (a) $P$, (b) $E(B-V)$, and (c) $P / E(B-V)$ versus $l$ for the stars with $P>0.1$ per cent at all $R$ for the same data as in Fig. 15 .

The dependences in Fig. 16 (a) and (b) are defined by the increases of both $P$ and $E(B-V)$ from all the data sources (i) about the direction to the Galactic centre, as well as in the Gould Belt about its (ii) higher $\left(l \approx 15^{\circ}, b \approx 19^{\circ}\right)$ and (iii) lower $\left(l \approx 195^{\circ}, b \approx-19^{\circ}\right)$ deviations from the Galactic mid-plane (see Gontcharov 2012b, Table 4). This results in two wide smooth increases: a higher one at $l \approx 0^{\circ}$ and 


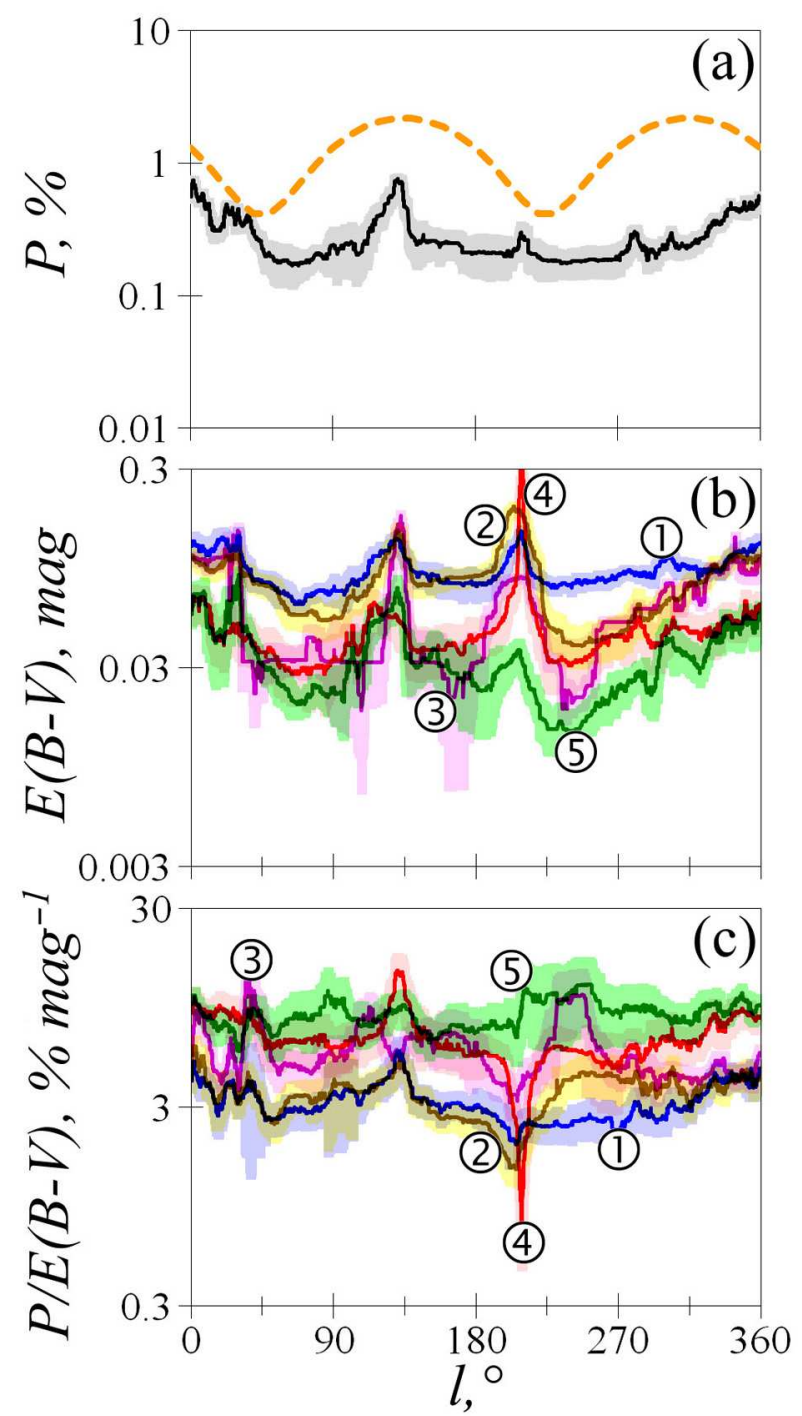

Figure 16. The same as Fig. 15 but for the stars with $P>0.1$ per cent at all the distances, and $l$ as the argument. The orange dashed curve is a function found by Fosalba et al. (2002).

a lower one at $l \approx 180^{\circ}$. This is seen in all reliable local reddening maps and models, at least, beginning with Arenou et al. 1992 (see, for example, Figure 2 in Gontcharov (2009)). Moreover, a non-uniform distribution of our sample makes some regions of the Belt more pronounced. For example, a lot of stars of the Perseus-Cassiopea $\left(l \approx 130^{\circ}, b \approx 0^{\circ}\right)$ and Orion $\left(l \approx 208^{\circ}, b \approx-19^{\circ}\right)$ complexes in the sample give the related spikes in Fig. 16.

A double sinusoid obtained by Fosalba et al. (2002) is shown in Fig. 16 by the orange dashed curve. Its extrema do not fit our data due to the consideration by Fosalba et al. of many stars with $R>500 \mathrm{pc}$, i.e. behind the Belt.

As Fig. 14 and 15, Fig. 16 shows that some large variations of $P$ and $E(B-V)$ with $l$ almost completely compensate each other, resulting in a nearly constant $P / E(B-V)$, albeit at some different levels for different reddening data sources.

\section{CONCLUSIONS}

We have analysed an all-sky compilation of the precise optical interstellar polarisation data from thirteen data sources for 3871 Gaia DR2 and Hipparcos stars within 500 pc from the Sun and shown them versus five 3D maps and models of reddening $E(B-V)$, the most reliable ones within this space. We excluded from consideration the stars with a known or suspected intrinsic polarisation. The comparison of the polarisation data from different data sources for common stars has shown that these data can be compiled in one sample.

We have created a map, where the median $P$ is given for a grid of 8 Galactic octants per 2 hemispheres per 2 latitude zones per 4 distance ranges within $500 \mathrm{pc}$. The value $P=0.1$ per cent can be accepted as a value which separates the space inside the Local Bubble with a lower $P$ and the space outside with a higher one. $P$ is maximal at the mid-plane of the Gould Belt, but not at the Galactic mid-plane.

In the Bubble, $P / R$ shows the same drop by about 6 times, as the drop of the volume density of $\mathrm{Na}$ I and neutral gas. In contrast, all the reddening data sources, except LVV, show a negligible drop of $E(B-V) / R$ and, hence, of dust volume density, which is similar to the drop by about 1.5 times of the volume density of Ca II. Thus, the Bubble seems to differ from the surrounding space by slightly lower volume density of dust and several times lower volume density of neutral gas. An overall gas-to-dust ratio in the Bubble can be maintained due to a several times higher volume density of ionised gas. Finally, it seems that the Bubble is not a space of a lower volume density of matter, but the one of a higher ionisation of it.

In contrast to the other reddening data sources, LVV shows in the Bubble a drop of $E(B-V) / R$ by an order of magnitude, i.e. comparable with the drop of $P / R$. Consequently, LVV gives $P / E(B-V)$ higher in- than outside the Bubble, while the remaining reddening data sources - vice versa. This result of LVV strongly contradicts to the chaotic $\theta$ in the Bubble.

Our analysis shows that the addition of a nearly constant systematic offset would eliminate most discrepancies between LVV and the other reddening data sources.

The 3D reddening map of G17 and 3D extinction model by G12 give an acceptable percentage of stars overcoming the Serkowski's limit $P / E(B-V)<9.3$ per cent per mag. The $3 \mathrm{D}$ map/models by AGG, DCL, and LVV give too many cases with $P / E(B-V)>9.3$ per cent per mag, leading us to the conclusion that either Serkowski's limit is not valid at all, or these maps/models considerably underestimate reddening within some regions. These regions mostly have $R<220$, $<150$ and $>250 \mathrm{pc}$ for DCL, LVV, and AGG, respectively. For AGG these regions are related to the partitioning of the sky during the creation of this model.

We found 67 stars with $P / E(B-V)>9.3$ per cent per mag based on the reddening estimates from all five data sources. These stars tend to concentrate near the Galactic centre, the Markkanen's cloud, the North Polar Spur, and some filament dust clouds. Probably, $P / E(B-V)$ is high for these stars due to an unusual extinction law, an underestimation of $E(B-V)$ in these regions, or an influence of oblate dust grains.

Outside the Bubble, the spatial variations of $P$ and its variations with dereddened colour $\left(G_{B P}-G_{R P}\right)_{0}$ are almost 
compensated by the variations of $E(B-V)$, resulting in a nearly constant $P / E(B-V)$. Hence, the well-known higher values of $P$ for $\mathrm{OB}$ and $\mathrm{M}$ stars are completely compensated by their higher $R$ and $E(B-V)$.

We found a chaotic behaviour of $\theta$ inside the Bubble. In contrast, the middle and high northern latitudes outside the Bubble are dominated by a giant envelope of aligned dust with a length of $>120^{\circ}$, a width of $>50^{\circ}$, and a depth of $>300$ pc. This envelope is elongated between $l \approx 40^{\circ}$ and $250^{\circ}$, i.e. nearly along the Local interstellar tunnel. The well-known Markkanen's cloud of aligned dust and the North Polar Spur belong to this envelope, while the two giant loops of aligned dust in the second and fourth quadrants seem to edge the envelope. All these structures are found as some emission/reddening filaments on the SFD map. Probably, this giant northern envelope continues at the low and southern latitudes.

\section{ACKNOWLEDGEMENTS}

We thank the reviewers Dr. Barry Welsh and Dr. Ralf Siebenmorgen for their useful comments. We thank Dr. Andrei Berdyugin for a discussion of his valuable polarisation results. We thank Dr. Ruslan Yudin and Dr. Vladimir Grinin for useful discussion of our results. The resources of the Centre de Données astronomiques de Strasbourg, Strasbourg, France (http://cds.u-strasbg.fr), including the SIMBAD database, were widely used in this study. This work has made use of data from the European Space Agency (ESA) mission Hipparcos. This work has made use of data from the European Space Agency (ESA) space mission Gaia (https://www.cosmos.esa.int/gaia), processed by the Gaia Data Processing and Analysis Consortium (DPAC, https://www . cosmos.esa.int/web/gaia/dpac/consortium). This research has made use of the Washington Double Star Catalog maintained at the U.S. Naval Observatory.

\section{REFERENCES}

Abt H., 2011, AJ, 141, 165

Alves F. O., Franco G. A. P., 2006, MNRAS, 366, 238

Andersson B-G, Potter S. B., 2007, ApJ, 665, 369

Arenou F., Grenon M., Gomez A., 1992, A\&A, 258, 104 (AGG)

Bailer-Jones C. A. L., 2015, PASP, 127, 994

Bailer-Jones C. A. L., Rybizki J., Fouesneau M., Mantelet G., Andrae R., 2018, AJ, 156, 58

Bailey J., Lucas P. W., Hough J. H., 2010, MNRAS, 405, 2570

Berdyugin A., Teerikorpi P., 2002, A\&A, 384, 1050

Berdyugin A. V., Teerikorpi P., Haikala L., Hanski M., Knude J., Markkanen T., 2001, A\&A, 372, 276

Berdyugin A., Piirola V., Teerikorpi P., 2014, A\&A, 561, A24

Bobylev V. V., 2014, Astrophysics, 57, 583

Bovy J., Rix H.-W., Green G. M., Schlafly E. F., D.P. Finkbeiner, 2016, ApJ, 818, 130

Bressan A., Marigo P., Girardi L., Salasnich B., Dal Cero C., Rubele S., Nanni A., 2012, MNRAS, 427, 127

Capitanio L., Lallement R., Vergely J.L., Elyajouri M., MonrealIbero A., 2017, A\&A, 606, A65

Cardelli J. A., Clayton G. C., Mathis J. S., 1989, ApJ, 345, 245

Cotton D. V., Bailey J., Kedziora-Chudczer L., Bott K., Lucas P. W., Hough J. H., Marshall J. P., 2016, MNRAS, 455, 1607
Cotton D. V., Marshall J. P., Bailey J., Kedziora-Chudczer L., Bott K., Marsden S. C., Carter B.D., 2017, MNRAS, 467, 873

Dotter A., 2016, ApJS, 222, 8

Drimmel R., Cabrera-Lavers A., López-Corredoira M., 2003, A\&A, 409, 205 (DCL)

FitzGerald M. P., 1968, AJ, 73, 983

Fosalba P., Lazarian A., Prunet S., Tauber J. A., 2002, ApJ, 564, 762

Frisch P.C., 2007, Space Sci. Rev., 130, 355

Frisch P.C. et al., 2015, ApJ, 814, 112

Gaia Collaboration et al., 2018a, A\&A, 616, A1

Gaia Collaboration et al., 2018b, A\&A, 616, A4

Gaia Collaboration et al., 2018c, A\&A, 616, A9

Girardi L., Groenewegen M.A.T., Hatziminaoglou E., da Costa L., 2005, A\&A, 436, 895

Gontcharov G. A., 2009, Astron. Lett., 35, 780

Gontcharov G. A., 2012a, Astron. Lett., 38, 12

Gontcharov G. A., 2012b, Astron. Lett., 38, 87 (G12)

Gontcharov G. A., 2016, Astrophysics, 59, 548

Gontcharov G. A., 2017, Astron. Lett., 43, 472 (G17)

Gontcharov G. A., Mosenkov A. V., 2017a, MNRAS, 470, L97

Gontcharov G. A., Mosenkov A. V., 2017b, MNRAS, 472, 3805

Gontcharov G. A., Mosenkov A. V., 2018, MNRAS, 475, 1121

Green G. M. et al., 2018, MNRAS, 478, 651

Heiles C., 2000, AJ, 119, 923

Høg E. et al., 2000, A\&A, 355, L27

Lallement R., Vergely J. L., Valette B., Puspitarini L., Eyer L., Casagrande L., 2014, A\&A, 561, A91 (LVV)

Lallement R., Snowden S., Kuntz K. D., Dame T. M., Koutroumpa D., Grenier I., Casandjian J. M., 2016, A\&A, 595,131

Leroy J. L., 1999, A\&A, 346, 955

Markkanen T., 1979, A\&A, 74, 201

Mason B. D., Wycoff G. L., Hartkopf W. I., Douglass G. G., Worley C.E., 2001, AJ, 122, 3466

Mathewson D. S., Ford V. L., 1970, Mem. RAS, 74, 139

McDonald I., Zijlstra A. A., Watson R. A., 2017, MNRAS, 471, 770

Medan I.,Andersson B-G, 2018, ApJ, in print

Meisner A. M., Finkbeiner D. P., 2015, ApJ, 798, 88

Michalik D., Lindegren L., Hobbs D., 2015, A\&A, 574, A115

Perryman M., 2009, Astronomical Applications of Astrometry: Ten Years of Exploitation of the Hipparcos Satellite Data. Cambridge Univ. Press, Cambridge, UK

Planck Collaboration et al., 2015, A\&A, 576, A106

Poidevin F., Bastien P., 2006, ApJ, 650, 945

Puspitarini L., Lallement R., 2012, A\&A, 545, A21

Reis W., Corradi W. J. B., 2008, A\&A, 486, 471

Reis W., Corradi W., de Avillez M. A., Santos F.P., 2011, ApJ, 734,8

Santos F. P., Corradi W., Reis W., 2011, ApJ, 728, 104

Schlegel D. J., Finkbeiner D. P., Davis M., 1998, ApJ, 500, 525 (SFD)

Serkowski K., 1974, in Carleton N., ed., Methods of Experimental Physics, Vol. 12: Astrophysics, Part A. Academic Press, New York, p. 361

Serkowski K., Mathewson D. S., Ford V. L., 1975, ApJ, 196, 261

Snowden S. L., Koutroumpa D., Kuntz K. D., Lallement R., Puspitarini L., 2015, ApJ, 806, 120

Stephens I. W., Looney L. W., Dowell C. D., Vaillancourt J. E., Tassis K., 2011, ApJ, 728, 99

van Leeuwen F., 2007, A\&A, 474, 653

Voshchinnikov N. V., Il'in V. B., Das H. K., 2016, MNRAS, 462, 2343

Welsh B., 1991, ApJ, 373, 556

Welsh B. Y., Sfeir D. M., Sirk M. M., Lallement R., 1999, A\&A, 352,308 
Welsh B. Y., Lallement R., Vergely J. L., Raimond S., 2010, A\&A, 510, A54

Wenger M. et al., 2000, A\&AS, 143, 9

Wills B. J., Wills D., Breger M., 2011, ApJS, 194, 19

Wright C. O., Egan M. P., Kraemer K. E., Price S. D., 2003, AJ, 125,359

Yudin R. V., 2001, A\&A, 368, 912

This paper has been typeset from a $\mathrm{T}_{\mathrm{E}} \mathrm{X} / \mathrm{LAT}_{\mathrm{E}} \mathrm{X}$ file prepared by the author. 